\title{
A new distribution-free generally weighted moving average monitoring scheme for detecting unknown shifts in the process location
}

\author{
Kutele Mabude $^{\mathrm{a}}$, Jean-Claude Malela-Majika ${ }^{\mathrm{a}}$ and Sandile Charles Shongwe $^{\mathrm{a}}$
}

${ }^{a}$ Department of Statistics, College of Science, Engineering and Technology, University of South Africa, P O Box 392 UNISA 0003 , Pretoria, South Africa

\begin{tabular}{l}
\hline C H R O N I C L E \\
\hline Article history: \\
Received July 152019 \\
Received in Revised Format \\
September 12019 \\
Accepted September 12019 \\
Available online \\
September 2019 \\
\hline Keywords: \\
Distribution-free \\
Time varying monitoring scheme \\
Asymptotic control limits \\
Exact control limits \\
Overall performance \\
Generally weighted moving \\
average
\end{tabular}

\section{Introduction}

A monitoring scheme is one of the most used tools in statistical process monitoring (SPM) to improve the process efficiency by identifying and controlling variability in order to achieve process stability. Monitoring schemes help to facilitate the identification of two types of variations in the process, namely, common (or chance) causes of variation and assignable (or special) causes of variation. A process that operates only in the presence of common causes is said to be in statistical control, or simply, in-control (IC). Otherwise, it is said to be out-of-control (OOC), see for example Montgomery (2005). An efficient monitoring scheme should be sensitive enough to detect small shifts in any type of process as quickly as possible. Time-weighted schemes such as the cumulative sum (CUSUM) and exponentially weighted

* Corresponding author

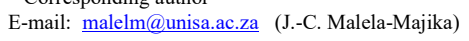

2020 Growing Science Ltd.

doi: $10.5267 /$ j.ijiec.2019.9.001 
moving average (EWMA) are developed to serve this purpose. Roberts (1959) introduced the EWMA monitoring scheme (denoted as $\bar{X}$-EWMA) to facilitate the detection of small shifts in the monitoring process. Since then, improving the sensitivity of the EWMA-based schemes has been the concern of many researchers, see the review by Ruggeri et al. (2007), and for other more recent improvements or enhancements, see for example, Haridy et al. (2019), Haq (2019), Adegoke et al. (2019), etc.

In an effort to further improve the EWMA-based schemes to monitor the process mean, Sheu and Lin (2003) proposed the generally weighted moving average (GWMA) scheme (denoted as $\bar{X}$-GWMA), which is a general version of both the EWMA scheme and the Shewhart-type scheme (this is shown in Section 2). They showed that the $\bar{X}$-GWMA scheme performs better than the $\bar{X}$-EWMA scheme in monitoring small shifts in the process mean. Thereafter, a number of researchers investigated the performance of parametric GWMA schemes, to count a few, Sheu and Yang (2006), Sheu and Hsieh (2009), Tai and Lin (2009), Teh et al. (2012), Aslam et al. (2017), Chakraborty et al. (2017), etc.

SPM schemes have been applied to a variety of fields, including engineering, production, manufacturing, finance, food industry, chemistry and biochemistry, see Simoglou et al. (1997), Black et al. (2011), Bag et al. (2012), Lim et al. (2017), etc. In practice, the underlying process distribution is generally unknown. In this case, control schemes that do not rely on parametric assumptions are needed. The foregoing GWMA monitoring schemes are based on the assumption of normality or some other underlying parametric distribution. However, when the data depart from normality, the performance of the $\bar{X}$ GWMA or any other parametric scheme degrades considerably. To remedy this problem, nonparametric GWMA schemes are recommended. Lu (2015) proposed a nonparametric GWMA monitoring scheme based on the sign statistic (denoted as SN-GWMA). Lu (2015) showed that the nonparametric SNGWMA scheme is more sensitive than the parametric $\bar{X}$-GWMA scheme under normal and a variety of other non-normal distributions. The GWMA scheme based on the signed-rank test (SR-GWMA) was proposed by Chakraborty et al. (2016) where, they showed that the SR-GWMA scheme performs better than the $\bar{X}$-GWMA, SN-GWMA and SN-EWMA schemes in many cases. More recently, Sukparungsee (2018) investigated the robustness of the SR-GWMA control scheme for monitoring the location shift of skew processes. Chakraborty et al. (2018) proposed a robust GWMA exceedance chart (EX-GWMA) for monitoring the location parameter. The quality of these monitoring schemes is found in their abilities to solve a variety of problems encountered in different environments. Two major problems faced in industrial and non-industrial processes are addressed in this paper. These problems are: (i) The assumption of normality is more questionable in industrial and non-industrial processes, and (ii) Most of the existing monitoring schemes are able to efficiently monitor either small shifts only, or large shifts only in the process parameters. Therefore, there is a need for more efficient and robust monitoring schemes that are able to detect small to large shifts in the process parameters without any distributional assumptions. Consequently, in this paper, we propose a new distribution-free GWMA control scheme based on one of the most powerful nonparametric tests (i.e. the Wilcoxon rank-sum (WRS) test) denoted as W-GWMA. The combination of the GWMA procedure and the WRS statistic enables the resulting scheme to efficiently monitor small and moderate shifts without affecting the performance of the monitoring scheme for large shifts.

The remainder of this paper is organised as follows: Section 2 provides the properties of the proposed W-GWMA scheme. Section 3 investigates the robustness and performance of the proposed monitoring scheme using extensive Monte Carlo simulations. Moreover, the W-GWMA scheme is compared to other existing time varying monitoring schemes. A real-life example is given in Section 4 to illustrate the implementation of the W-GWMA scheme. In Section 5, the effect of the estimation of design parameters and Phase I sample size on the IC and OOC Phase II performances of the proposed W-GWMA scheme is investigated using its conditional run-length distribution. Concluding remarks and recommendations are given in Section 6. 


\section{Design of the proposed monitoring schemes}

Assume that $X=\left\{x_{i}, i=1,2, \ldots, m\right\}$ represents the IC Phase I (or reference) sample with unknown or non-normal continuous cumulative distribution function (cdf) $F(x)$ and $Y=\left\{y_{t j}, j=1,2, \ldots, n ; t=1\right.$, $2, \ldots\}$ represents the Phase II (or test) sample with cdf $G(y)$. The test samples at time $t(t=1,2, \ldots)$ are assumed to be independent and identically distributed (iid) from each other and from the reference sample. Let $F(t)=G(t-\delta)$, for all $t$, where $\delta$ is the change (or shift) in the location parameter. The process is then considered IC if $\delta=0$, which means $F(t)=G(t) \forall t$.

\subsection{The Wilcoxon rank-sum statistic}

The Wilcoxon rank-sum (WRS) for two-sample test proposed by Wilcoxon (1945) is defined by

$$
W_{t}=\sum_{s=1}^{m+n}\left(s \cdot x_{(s)}^{\prime}\right), t=1,2,3, \ldots
$$

where $x_{(s)}^{\prime}$ are the ordered observations obtained after combining the reference and test sample and arranging the $(m+n)$ observations in ascending order. Note that $x_{(s)}^{\prime}=1$ if $x_{s}^{\prime}$ comes from the test sample and $x_{(s)}^{\prime}=0$ if $x_{s}^{\prime}$ comes from reference sample. The mean and variance of the $W$ statistic under the identical distributions assumption are, respectively, given by

$$
E\left(W_{t}\right)=\mu_{W}=\frac{n(m+n+1)}{2}
$$

and

$$
\operatorname{Var}\left(W_{t}\right)=\sigma_{W}^{2}=\frac{m n(m+n+1)}{12} .
$$

The above measures are very useful in the design and implementation of the W-GWMA monitoring scheme as well as Li et al. (2010)'s EWMA scheme.

\subsection{The Proposed $W-G W M A$ monitoring scheme}

Following Sheu and Lin (2003)'s idea, the charting statistic of the W-GWMA monitoring scheme, denoted $G E_{t}$, is given by

$$
G E_{t}=P(M=1) W_{t}+P(M=2) W_{t-1}+\cdots+P(M=t) W_{1}+P(M>t) W_{0},
$$

with

and

$$
P(M=t)=q^{(t-1)^{\alpha}}-q^{t^{\alpha}}
$$

$$
P(M>t)=q^{t^{\alpha}},
$$

where $M$ is the number of samples until the first occurrence of event $A$ since the previous occurrence of event $A, P(M=1)$ represents the weight value for the current sample, $P(M=2)$ is the weight value for the previous sample, $P(M=t)$ is the weight value for the first sample and $P(M>t)$ is the weight value for the target value of the process mean, which is considered to be the unconditional IC expectation of $W_{t}$ given by $W_{0}=E\left(W_{t} \mid I C\right)=\mu_{W}$. The design parameter $q(0 \leq q<1)$ is constant and the adjustment parameter $\alpha(\alpha>0)$ is determined by the practitioners.

Following a similar procedure as Sheu and Lin (2003) and Sheu and Hsieh (2009), it can be shown without loss of generality that the W-GWMA statistic in Eq. (3) can be written as 


$$
G E_{t}=\sum_{i=1}^{t}\left(q^{(i-1)^{\alpha}}-q^{i^{\alpha}}\right) W_{t-i+1}+q^{t^{\alpha}} \mu_{W}, \quad t=1,2,3, \ldots
$$

The expected value of Eq. (4) is given by

$$
E\left(G E_{t}\right)=E\left[\sum_{i=1}^{t}\left(q^{(i-1)^{\alpha}}-q^{i^{\alpha}}\right) W_{t-i+1}+q^{t^{\alpha}} \mu_{W}\right]=\mu_{W}=\frac{n(m+n+1)}{2} .
$$

The variance of Eq. (4) is then defined by

$$
\operatorname{Var}\left(G E_{t}\right)=\sum_{i=1}^{t}\left(q^{(i-1)^{\alpha}}-q^{i^{\alpha}}\right)^{2} \operatorname{Var}\left(W_{t-i+1}\right) .
$$

Eq. (6) can be written as

$$
\operatorname{Var}\left(G E_{t}\right)=\sigma_{G E}^{2}=Q_{t} \sigma_{W}^{2}
$$

where

$$
Q_{t}=\sum_{i=1}^{t}\left(q^{(i-1)^{\alpha}}-q^{i^{\alpha}}\right)^{2}
$$

and

$$
\sigma_{W}^{2}=\frac{m n(m+n+1)}{12} .
$$

Therefore, the exact (hereafter Case E) control limits of the proposed W-GWMA monitoring scheme can be calculated as

$$
\begin{aligned}
& L C L_{G E}=\mu_{W}-L_{G E} \sigma_{G E} \\
& C L_{G E}=\mu_{W} \\
& U C L_{G E}=\mu_{W}+L_{G E} \sigma_{G E}
\end{aligned}
$$

where $L_{G E}$ (with $L_{G E}>0$ ) represents the W-GWMA monitoring scheme coefficient. This coefficient is used to fix the predefined nominal IC average run-length $(A R L)$ value. The W-GWMA scheme is constructed by plotting the charting statistic $G E_{t}$ against the sampling time (or sample number) $t$. The process is considered to be $\mathrm{OOC}$ if $G E_{t}$ falls beyond the control limits, that is, $G E_{t} \geq U C L_{G E}$ or $G E_{t} \leq$ $L C L_{G E}$; otherwise, the process is considered to be IC.

Note that when $\alpha=1$, it can be shown that

$Q_{t}=\left(1-q^{2 t}\right) \frac{1-q}{1+q}$,

so that Eq. (7) reduces to

$$
\operatorname{Var}\left(G E_{t}\right)=\sigma_{G E}^{2}=\left(1-q^{2 t}\right) \frac{1-q}{1+q} \sigma_{W}^{2} .
$$

Therefore, when the process has been running for a long time, that is, when $t$ tends to infinity $(t \rightarrow \infty)$ then $q^{2 t} \rightarrow 0$. Then, the variance of the W-GWMA statistic becomes 


$$
\operatorname{Var}\left(G E_{t}\right)=\sigma_{G E}^{2}=m n \frac{1-q}{1+q}\left(\frac{m+n+1}{12}\right) .
$$

Therefore, the control limits based on Eq. (10) are called asymptotic (hereafter Case A) control limits; whereas, those based on Eq. (7) are called time varying (hereafter Case E) control limits. Note that when $1-q=\lambda$ (i.e., $q=1-\lambda$ ) and $\alpha=1$, the W-GWMA scheme is equivalent to Li et al. (2010)'s WEWMA scheme; however, when $q=0$ and $\alpha=1$, the GWMA scheme is equivalent to the Shewhart WRS scheme (denoted as W-Shewhart).

In this paper, the proposed W-GWMA monitoring scheme with design parameters $q$ and $\alpha$ will be denoted as W-GWMA $(q, \alpha)$; while Li et al. (2010)'s EWMA scheme will be denoted as W-EWMA $(\lambda)$ where $\lambda$ is the smoothing parameter of the W-EWMA scheme.

\section{Performance study}

\subsection{Performance measures}

To evaluate the performance of a monitoring scheme, the literature recommends the use of the $A R L$ value. This value represents the mean of the run-length distribution, which is the number of rational subgroups to be plotted before the monitoring scheme signals for the first time. A number of authors have criticised the sole use of this measure for two main reasons, which are: (i) the $A R L$ value does not give enough information since the run-length distribution is highly skewed, and (ii) the $A R L$ value assesses the performance of a monitoring scheme for a specific shift (Teh et al., 2014; Shongwe \& Graham, 2017). In practice, we need to get useful information missing in the $A R L$ criterion and assess the performance over a range of shifts including the overall performance of a monitoring scheme. To solve these problems, the SPM literature suggests the use of the percentiles of the run-length $(P R L)$ and the characteristics of the quality loss function $(Q L F)$ such as the average extra quadratic loss $(A E Q L)$ values as performance measures. In this paper, the $A R L$, standard deviation of the run-length $(S D R L), P R L$ and $A E Q L$ values are used to evaluate the performance of the proposed W-GWMA $(q, \alpha)$ monitoring scheme. The $A E Q L$ is the quadratic weighted mean of the $A R L$ value over the range of shifts $\delta_{\min } \leq \delta \leq \delta_{\max }$. Therefore, the $A E Q L$ value is defined by

$$
A E Q L=\frac{1}{\delta_{\max }-\delta_{\min }} \int_{\delta_{\min }}^{\delta_{\max }} \delta^{2} A R L(\delta) d \delta .
$$

Eq. (11) can also be written as:

$$
A E Q L=\frac{1}{\delta_{\max }-\delta_{\min }} \sum_{\delta=\delta_{\min }}^{\delta_{\max }} \delta^{2} A R L(\delta) .
$$

where $A R L(\delta)$ is the OOC $A R L$ for a specific mean shift $(\delta)$. In this paper, we use a step shift of size 0.1 .

In addition to the $A E Q L$ value, the average ratio of the average run-length ( $A R A R L)$ and the performance comparison index $(P C I)$ values are used as overall performance measures. The ARARL and PCI are mathematically defined by

$$
A R A R L=\frac{1}{\delta_{\max }-\delta_{\min }} \sum_{\delta=\delta_{\min }}^{\delta_{\max }} \frac{A R L(\delta)}{A R L(\delta)_{\text {benchmark }}}
$$

and

$$
P C I=\frac{A E Q L}{A E Q L_{\text {benchmark }}},
$$


respectively, where the benchmark scheme is chosen to be the monitoring scheme with a minimum $A E Q L$ value. If the $A R A R L$ is greater than one, the corresponding scheme is less efficient than the benchmark scheme over the range of shifts under consideration.

\subsection{Design considerations}

The optimal design parameters are found by solving the following optimization model:

$\operatorname{Min}_{L, q, \alpha} A E Q L$

subject to

$A R L_{0} \in S$, with $S=[\tau-\xi \tau, \tau+\xi \tau]$,

where $A R L_{0}$ is the attained IC $A R L$ value, $\tau$ represents the predefined nominal $A R L_{0}$ and generally, $\xi$ is taken to be equal to 0.1 . The value of $\tau$ is set to be equal to some high desired value such as 250,370 and 500 .

Therefore, the optimal W-GWMA $(q, \alpha)$ monitoring scheme is designed as follows:

Step 1 Specify the monitoring scheme parameters (i.e. $q$ and $\alpha$ ) as well as the distribution parameters and the nominal $A R L_{0}$ value (i.e., $\tau$ ).

Step 2 Initialize the variable $A E Q L$ to a very large value, say $10^{5}$, used as the initial minimum value of the $A E Q L$ values denoted by $A E Q L_{\text {min }}$.

Step 3 Search for the value of $L$ (i.e., $L_{G E}$ ) for which the attained $A R L_{0}$ of the proposed W-GWMA monitoring scheme is very close or equal to $\tau$; then go to Step (4). If this does not happen, then go to Step (6).

Step 4 Compute the OOC $A R L\left(A R L_{\delta}\right)$ values and calculate the corresponding $A E Q L$ value denoted $A E Q L_{\text {cal }}$.

Step 5 Compare the $A E Q L_{\text {cal }}$ to the current $A E Q L_{\text {min }}$. If $A E Q L_{\text {cal }}<A E Q L_{\text {min }}$ then the current $A E Q L_{\text {min }}=A E Q L_{\text {cal }}$. Otherwise, current $A E Q L_{\text {min }}=A E Q L_{\text {min }}$. The parameters corresponding to the current $A E Q L_{\text {min }}$ are recorded as current parameters then return to Step (3).

Step 6 The current parameters are recorded as optimal parameters corresponding to the optimal monitoring scheme with a minimum $A E Q L$ value. The design of the optimal W-GWMA $(q, \alpha)$ is completed.

Note that in Step (3) the attained $A R L_{0}$ value is considered very close to $\tau$ if $A R L_{0} \in S$ with $S=\tau \pm \xi \tau$, where $\xi=0.04$, as it provides more accuracy as compared to the traditional value of 0.1 . For instance, when $\tau=500$, the $A R L_{0}$ is considered very close to $\tau$ if $A R L_{0} \in[480,520]$.

Readers are referred to Malela-Majika et al. (2016) and Li et al. (2010) for more information on the computation of the $A R L$ values using extensive simulations.

\subsection{In-control performance and robustness of the proposed W-GWMA monitoring scheme}

A monitoring scheme is said to be IC robust if the IC characteristics of the run-length distribution (such as the $A R L_{0}$, the IC median run-length $\left(M R L_{0}\right)$, etc.) are the same over all continuous distributions. To check this, we have computed the IC characteristics of the run-length distribution under symmetrical and skewed distributions. In this paper, we considered the following five distributions:

(i) Standard normal distribution, denoted $N(0,1)$,

(ii) Student's $t$ distribution with degrees of freedom $v=10$, denoted $t(10)$,

(iii) Gamma distribution with parameters $\omega=3$ and $\beta=1$, denoted $\operatorname{GAM}(3,1)$,

(iv) Log-logistic distribution with parameters $\omega=1$ and $\beta=3$, denoted $\log L(1,3)$

(v) Weibull distribution with parameters $\kappa=2$ and $\varsigma=1$, denoted $\operatorname{Weib}(2,1)$. 
For a fair comparison, the above distributions are transformed such that the mean and variance are equal to 0 and 1 , respectively.

Tables 1 and 2 give the Case $\mathrm{A}$ and Case $\mathrm{E}$ attained $A R L_{0}$ and $A E Q L$ values of the proposed W$\operatorname{GWMA}(q, \alpha)$ scheme when $n \in\{3,5\}, m \in\{50,100,500\}, q \in\{0.1,0.5,0.7,0.9\}$ and $\alpha \in\{0.5,1$, $1.5\}$ for a nominal $A R L_{0}$ of 500 under different probability distributions. The results in Tables 1 and 2 show that for both Case A and Case E, the width of the control limits widens as the Phase I sample size increases. For instance, in Case A, when $(m, n)=(50,3)$, we found that $L=3.0646$ so that the W-GWMA $(0.1,0.5)$ yields and attained $A R L_{0}$ of 501.84 under the $N(0,1)$ distribution. However, when $(m, n)=(500$, 3 ), we found that $L=3.2973$ so that the W-GWMA $(0.1,0.5)$ yields an attained $A R L_{0}$ of 502.61 under the same distribution (see Table 1). When the Phase II (or test) sample increases, the width of the control limits broadens so that the proposed scheme yields an attained $A R L_{0}$ as close as possible to 500. For instance, when $(m, n)=(50,3)$, we found that $L=3.1232$ so that the proposed W-GWMA $(0.1,1)$ yields an $A R L_{0}$ of 503.74. However, when $(m, n)=(50,5)$, we found that $L=3.1877$ so that the proposed W-GWMA $(0.1$, 1) yields an $A R L_{0}$ of 499.11. For a pre-specified $A R L_{0}$ value, we can also see that when $\alpha$ is kept fixed, as $q$ increases, the width of the control limits narrows. However, when $q$ is kept constant, as $\alpha$ increases, the width of the control limits broadens.

It is very important to report that for both Case $\mathrm{A}$ and Case $\mathrm{E}$, the attained $A R L_{0}$ values are much closer to the nominal $A R L_{0}$ value of 500 across all continuous probability distributions for each set of optimal parameters. For instance, in Case A, when $(m, n)=(100,5)$ and $(q, \alpha, L)=(0.7,0.5,2.8240)$, the attained $A R L_{0}$ values obtained from the proposed W-GWMA $(0.7,0.5)$ scheme under the $N(0,1), t(10), \operatorname{GAM}(3,1)$, $\log L(1,3)$ and $\operatorname{Weib}(2,1)$ are equal to $499.86,501.56,510.66,497.46$ and 508.78, respectively. This shows that the proposed W-GWMA $(q, \alpha)$ monitoring scheme is IC robust. From both Tables 1 and 2, it can also be seen that when $\alpha=1$, the proposed W-GWMA $(q, \alpha)$ scheme is equivalent to Li et al. (2010)'s W$\operatorname{EWMA}(\lambda)$ scheme with $\lambda=1-q$ (i.e., W-GWMA $(q, 1)$ scheme $\equiv \mathrm{W}-\operatorname{EWMA}(1-q)$ scheme). Note that the trend of the findings remains valid for other prespecified nominal $A R L_{0}$ values such as $250,370,1000$, etc. Therefore, in this paper we will focus on investigating the performance of the proposed W$\operatorname{GWMA}(q, \alpha)$ scheme for a nominal $A R L_{0}$ value of 500 and $(m, n)=(100,5)$.

Given that the W-GWMA $(q, \alpha)$ scheme is IC robust, the optimal parameters may now be used to investigate the $\mathrm{OOC}$ performance of the proposed monitoring scheme.

\subsection{OOC Performance of the $W$-GWMA monitoring scheme}

In this section, we discuss the OOC performance (see Tables 3-5) as well as the overall performance of the proposed monitoring scheme (see Tables 1 and 2 - second row). Tables 1 and 2 do not only investigate the IC robustness of the proposed scheme (see first row of each cell in Tables 1 and 2), they also present the overall performance of the proposed scheme for different reference and test sample sizes (i.e. different $m$ and $n$ values) under different distributions. The second row of each cell in Tables 1 and 2 gives the $A E Q L$ values of the proposed control scheme for different design parameters. However, Tables 3-5 display the OOC characteristics (or properties) of the run-length distribution under different distributions for both Case A and Case E when $(m, n)=(100,5)$. The first row of each cell in Tables 3-5 gives the ARL and SDRL values and the second row gives the $5^{\text {th }}, 25^{\text {th }}, 50^{\text {th }}, 75^{\text {th }}$ and $95^{\text {th }} P R L$ values of the W-GWMA monitoring schemes. Moreover, these characteristics are given along with some corresponding overall performance measures (i.e. ARARL and PCI) under different distributions. From Tables 1 and 2, we observed that as the Phase I sample size increases, the overall performance of the proposed scheme increases in terms of the $A E Q L$ values. For instance, under the $N(0,1)$ distribution, when $(m, n)=(50,3)$, the proposed W-GWMA $(0.1,0.5)$ scheme yields $A E Q L$ values of 132.79 and 134 in Case A and Case E, respectively. However, when $(m, n)=(500,3)$, the proposed W-GWMA $(0.10,0.5)$ scheme yields $A E Q L$ values of 110.88 and 109.83 in Case A and Case E, respectively. 
Table 1

Case A attained $A R L_{0}$ (first row) and $A E Q L$ (second row) of the proposed W-GWMA control scheme when $n \in\{3,5\}, m \in\{50,100,500\}, q \in\{0.1,0.5,0.7$,

$0.9\}$ and $\alpha \in\{0.5,1,1.5\}$ for a nominal $A R L_{0}$ of 500 under different probability distribution functions

\begin{tabular}{|c|c|c|c|c|c|c|c|c|c|c|c|c|c|c|c|c|c|c|c|c|}
\hline \multirow[b]{2}{*}{$n$} & \multirow[b]{2}{*}{$q$} & \multirow[b]{2}{*}{$\alpha$} & \multicolumn{6}{|c|}{$\boldsymbol{m}=\mathbf{5 0}$} & \multicolumn{6}{|c|}{$m=100$} & \multicolumn{6}{|c|}{$m=\mathbf{5 0 0}$} \\
\hline & & & $L$ & $N(0,1)$ & $t(10)$ & $\operatorname{GAM}(\mathbf{3}, 1)$ & $\log L(1,3)$ & Weib $(2,1)$ & $L$ & $N(0,1)$ & $t(10)$ & $\operatorname{GAM}(3,1)$ & $\log L(1,3)$ & Weib $(2,1)$ & $L$ & $N(0,1)$ & $t(10)$ & $\operatorname{GAM}(3,1)$ & $\log L(1,3)$ & Weib $(2,1)$ \\
\hline & \multirow[t]{6}{*}{0.1} & 0.5 & 3.0646 & 501.84 & 498.11 & 492.05 & 499.69 & 492.57 & \multirow{2}{*}{3.2548} & 495.35 & 495.94 & 493.6 & 499.68 & 489.69 & \multirow{2}{*}{3.2973} & 502.61 & 496.13 & 496.86 & 498.55 & 496.19 \\
\hline & & \multirow{3}{*}{1} & & 132.79 & 110.19 & 237.11 & 167.98 & 188.14 & & 119.62 & 89.09 & 175.86 & 105.12 & 154.76 & & 110.88 & 77.93 & 147.83 & 84.18 & 137.16 \\
\hline & & & 3.1232 & 503.74 & 503.28 & 506.38 & 501.67 & 493.79 & \multirow[t]{2}{*}{3.2860} & 506.01 & 504.03 & 509.12 & 517.09 & 530.26 & \multirow[t]{2}{*}{3.3393} & 501.95 & 497.14 & 497.72 & 515.29 & 500.38 \\
\hline & & & & 136.23 & 112.04 & 241.42 & 172.19 & 197.65 & & 122.44 & 90.19 & 186.54 & 110.38 & 160.34 & & 111.58 & 78.68 & 149.85 & 84.95 & 139.47 \\
\hline & & \multirow[t]{2}{*}{1.5} & 3.0864 & 517.73 & 512.21 & 507.5 & 490.83 & 495.35 & \multirow[t]{2}{*}{3.2686} & 496.21 & 490.61 & 494.05 & 493.81 & 492.31 & 3.3088 & 514.63 & 497.98 & 514.76 & 515.05 & 496.82 \\
\hline & & & & 136.80 & 111.18 & 247.82 & 171.79 & 192.28 & & 124.87 & 92.19 & 187.08 & 110.93 & 159.2 & & 112.58 & 98.81 & 151.56 & 85.37 & 141.18 \\
\hline & 0.5 & 0.5 & 2.8073 & 514.06 & 507.73 & 511.47 & 510.92 & 491.29 & 2.9301 & 494.11 & 499.51 & 488.81 & 508.35 & 488.44 & 2.9398 & 501.52 & 498.06 & 500.73 & 507.05 & 502.37 \\
\hline & & & & 113.43 & 90.12 & 131.4 & 90.68 & 129.92 & & 102.02 & 85.09 & 112.08 & 84.8 & 112.5 & & 93.11 & 76.59 & 101.23 & 81.71 & 101.46 \\
\hline & & 1 & 2.9230 & 490.39 & 493.27 & 484.27 & 478.04 & 482.43 & 2.9896 & 500.1 & 493.61 & 487.84 & 489.68 & 509.1 & 3.1869 & 492.1 & 502.83 & 509.24 & 494.27 & 506.66 \\
\hline & & & & 123.37 & 102.3 & 139.99 & 100.4 & 137.53 & & 115.22 & 96.84 & 119.89 & 91.29 & 122.01 & & 109.08 & 93.46 & 107.3 & 83.66 & 111.42 \\
\hline & & 1.5 & 2.9105 & 511.85 & 494.64 & 523.05 & 490.82 & 514.91 & 2.9602 & 497.24 & 491.85 & 505.98 & 499.94 & 498.76 & 3.0953 & 496.22 & 498.99 & 497.26 & 492.3 & 495.6 \\
\hline & & & & 130.55 & 105.48 & 152.45 & 108.27 & 147.45 & & 126.94 & 99.17 & 128.18 & 95.22 & 130.4 & & 113.22 & 95.2 & 114.29 & 89.72 & 117.99 \\
\hline 3 & 0.7 & 0.5 & 2.6364 & 495.16 & 512.79 & 493.91 & 475.95 & 518.86 & 2.6525 & 506.26 & 508.94 & 508.79 & 501.2 & 499.15 & 2.7364 & 493.5 & 503.01 & 501.16 & 495.72 & 499.2 \\
\hline & & & & 111.19 & 90.02 & 126.95 & 90.79 & 128.65 & & 103.9 & 86.51 & 111.87 & 89.14 & 116.36 & & 92.54 & 73.15 & 100.52 & 80.89 & 100.66 \\
\hline & & 1 & 2.7222 & 492.5 & 506.99 & 527.6 & 515.12 & 501.72 & 2.7404 & 506.63 & 496.3 & 501.16 & 497.83 & 494.13 & 2.8300 & 496.71 & 507.91 & 507.76 & 489.91 & 488.91 \\
\hline & & & & 120.82 & 100.9 & 126.67 & 95.13 & 128.08 & & 113.22 & 96.69 & 110.65 & 88.57 & 115.2 & & 106.16 & 93.49 & 101.69 & 82.19 & 105.8 \\
\hline & & 1.5 & 2.6674 & 495.77 & 488.12 & 507.36 & 503.5 & 491.26 & 2.6774 & 488.23 & 495.2 & 497.85 & 487.23 & 492.73 & 2.7855 & 502.67 & 512.67 & 505.27 & 511.36 & 512.54 \\
\hline & & & & 123.72 & 102.13 & 142.09 & 97.97 & 134.87 & & 115.81 & 97.09 & 116.42 & 90.12 & 119.7 & & 108.74 & 94.22 & 105.51 & 86.94 & 110.10 \\
\hline & 0.9 & 0.5 & 2.4029 & 492.73 & 492.47 & 490.64 & 505.88 & 485.24 & 2.5049 & 510.25 & 508.89 & 499.95 & 510.78 & 511.9 & 2.6807 & 504.64 & 503.86 & 498.97 & 507.57 & 504.13 \\
\hline & & & & 109.68 & 80.89 & 110.4 & 88.72 & 121.21 & & 103.61 & 80.09 & 105.08 & 89.04 & 117.73 & & 92.56 & 70.37 & 84.74 & 90.16 & 117.13 \\
\hline & & 1 & 2.5211 & 498.09 & 491.3 & 492.3 & 490.83 & 490.38 & 2.6214 & 498.64 & 507.35 & 507.45 & 508.31 & 490.75 & 2.7191 & 503.61 & 500.75 & 497.31 & 501.42 & 499.16 \\
\hline & & & & 119.51 & 96.22 & 118.56 & 92.85 & 122.39 & & 110.79 & 93.81 & 106.04 & 92.28 & 115.66 & & 102.75 & 93.24 & 105.63 & 85.97 & 101.24 \\
\hline & & 1.5 & 2.4762 & 504.92 & 504.82 & 506.45 & 496.13 & 488.69 & 2.5849 & 512.70 & 510.91 & 514.26 & 509.69 & 491.77 & 2.6989 & 497.89 & 496.85 & 496.31 & 489.32 & 502.06 \\
\hline & & & & 121.56 & 98.18 & 140.78 & 94.75 & 139.22 & & 117.70 & 97.08 & 113.9 & 92.89 & 118.27 & & 108.22 & 93.24 & 105.06 & 85.13 & 109.71 \\
\hline & 0.1 & 0.5 & 3.0725 & 508.51 & 499.81 & 491.82 & 504.03 & 504.05 & 3.1969 & 509.51 & 510.18 & 518.81 & 517.41 & 521.71 & 3.2705 & 493.21 & 502.1 & 501.98 & 495.43 & 498.59 \\
\hline & & & & 91.94 & 68.62 & 129.51 & 77.2 & 117.29 & & 84.54 & 60.90 & 101.06 & 60.36 & 99.94 & & 76.16 & 55.43 & 82.8 & 51.15 & 84.53 \\
\hline & & 1 & 3.1877 & 499.11 & 505.23 & 496.6 & 499.95 & 488.66 & 3.2029 & 501.63 & 499.42 & 509.12 & 496.33 & 514.7 & 3.3031 & 497.65 & 495.18 & 499.92 & 499.92 & 495.9 \\
\hline & & & & 94.19 & 69.39 & 130.19 & 81.57 & 118.75 & & 85.00 & 62.08 & 102.16 & 61.77 & 100.19 & & 77.65 & 57.34 & 84.14 & 53.14 & 85.9 \\
\hline & & 1.5 & 3.0979 & 491.53 & 488.13 & 481.12 & 481.6 & 487.16 & 3.1062 & 492.25 & 497.81 & 492.39 & 489.37 & 491.01 & 3.2878 & 493.13 & 483.4 & 488.01 & 493.02 & 508.12 \\
\hline & & & & 94.04 & 70.22 & 132.48 & 82.51 & 117.26 & & 86.06 & 63.41 & 103.08 & 62.36 & 100.94 & & 78.46 & 58.29 & 85.31 & 54.26 & 87.04 \\
\hline & 0.5 & 0.5 & 2.8228 & 483.5 & 490.23 & 503.66 & 497.65 & 506.38 & 2.8413 & 489.94 & 486.68 & 495.15 & 492.03 & 487.69 & 2.9986 & 501.1 & 503.74 & 492.94 & 496.99 & 491.06 \\
\hline & & & & 76.44 & 58.54 & 83.63 & 54.21 & 83.09 & & 68.29 & 54.01 & 67.38 & 47.33 & 70.59 & & 62.48 & 51.33 & 58.86 & 45.2 & 62.30 \\
\hline & & 1 & 2.9416 & 505.25 & 513.9 & 502.53 & 525.63 & 514 & 2.9516 & 503.2 & 516.4 & 505.99 & 502.93 & 525.62 & 3.0926 & 495.27 & 498.17 & 503.18 & 492.03 & 503.61 \\
\hline & & & & 84.42 & 63.2 & 95.32 & 62.93 & 94.68 & & 75.63 & 58.01 & 77.52 & 53.27 & 79.85 & & 68.27 & 54.28 & 66.00 & 48.49 & 69.00 \\
\hline & & 1.5 & 2.9331 & 515.31 & 527.31 & 503.96 & 517.85 & 491.06 & 2.9420 & 492.02 & 494.24 & 495.53 & 498.18 & 500.43 & 3.0680 & 496.93 & 495.4 & 492.71 & 495.59 & 495.10 \\
\hline & & & & 103.43 & 83.17 & 118.95 & 86.45 & 115.92 & & 91.3 & 76.36 & 100.60 & 78.01 & 100.46 & & 81.74 & 67.24 & 88.60 & 72.40 & 88.43 \\
\hline 5 & 0.7 & 0.5 & 2.8013 & 508.69 & 507.47 & 503.59 & 499.68 & 505.38 & 2.8240 & 499.86 & 501.56 & 510.66 & 497.46 & 508.78 & 2.9100 & 501.16 & 497.55 & 495.5 & 491.52 & 495.19 \\
\hline & & & & 81.31 & 62.07 & 89.57 & 58.2 & 87.61 & & 72.01 & 56.44 & 71.905 & 50.5 & 73.84 & & 64.63 & 52.94 & 61.12 & 46.89 & 64.1 \\
\hline & & 1 & 2.9525 & 520.21 & 507.05 & 503.41 & 543.7 & 485.77 & 2.9950 & 506.36 & 499.28 & 512.56 & 483.07 & 520.41 & 2.9988 & 494.86 & 514.25 & 492.75 & 503.35 & 497.3 \\
\hline & & & & 104.49 & 90.79 & 110.63 & 90.87 & 109.54 & & 97.21 & 87.4 & 95.85 & 83.82 & 98.11 & & 91.96 & 85.54 & 89.15 & 82.84 & 91.67 \\
\hline & & 1.5 & 2.8661 & 518.14 & 493.84 & 505.14 & 516.12 & 512.04 & 2.9254 & 484.05 & 496.77 & 502.42 & 504.39 & 499.35 & 2.9297 & 490.58 & 495.42 & 485.56 & 493.98 & 492.47 \\
\hline & & & & 106.84 & 91.93 & 112.68 & 93.53 & 114.26 & & 99.25 & 87.87 & 98.54 & 84.54 & 101.32 & & 93.36 & 85.84 & 90.76 & 83.07 & 93.42 \\
\hline & 0.9 & 0.5 & 2.7057 & 506.87 & 509.9 & 506.94 & 494.81 & 513.27 & 2.7283 & 515.43 & 497.74 & 495.75 & 514.11 & 507.57 & 2.7401 & 498.59 & 496.88 & 489.34 & 506.27 & 496.84 \\
\hline & & & & 109.87 & 95.63 & 110.79 & 88.48 & 114.82 & & 102.73 & 91.36 & 98.55 & 85.31 & 102.49 & & 95.81 & 88.49 & 92.14 & 83.91 & 94.97 \\
\hline & & 1 & 2.7341 & 485.98 & 481.44 & 494.26 & 497.75 & 486.74 & 2.9854 & 521.88 & 509.05 & 500.96 & 503.67 & 510.61 & 3.0043 & 495.77 & 489.08 & 496.33 & 493.1 & 503.64 \\
\hline & & & & 134.54 & 117.29 & 137.81 & 115.88 & 141.33 & & 122.05 & 108.18 & 123.8 & 107.16 & 125.05 & & 107.46 & 96.97 & 104.93 & 90.52 & 106.91 \\
\hline & & 1.5 & 2.7222 & 497.74 & 491.49 & 503.12 & 499.81 & 508.21 & 2.9086 & 500.39 & 510.14 & 504.96 & 500.42 & 511.57 & 2.9814 & 505.48 & 495.79 & 500.05 & 500.56 & 509.17 \\
\hline & & & & 141.702 & 129.6 & 147.32 & 126.63 & 146.73 & & 135.13 & 126.38 & 132.132 & 123.63 & 135.53 & & 127.02 & 122.91 & 127.37 & 122.79 & 128.91 \\
\hline
\end{tabular}

Note: When $\alpha=1$, the proposed W-GWMA $(q, \alpha)$ is equivalent to the W-EWMA $(\lambda)$ of Li et al. (2010) where $\lambda=1-q$. 
Table 2

Case E attained $A R L_{0}$ (first row) and $A E Q L$ (second row) of the proposed W-GWMA control scheme when $n \in\{3,5\}, m \in\{50,100,500\}, q \in\{0.1,0.5,0.7$, $0.9\}$ and $\alpha \in\{0.5,1,1.5\}$ for a nominal $A R L_{0}$ of 500 under different probability distribution functions

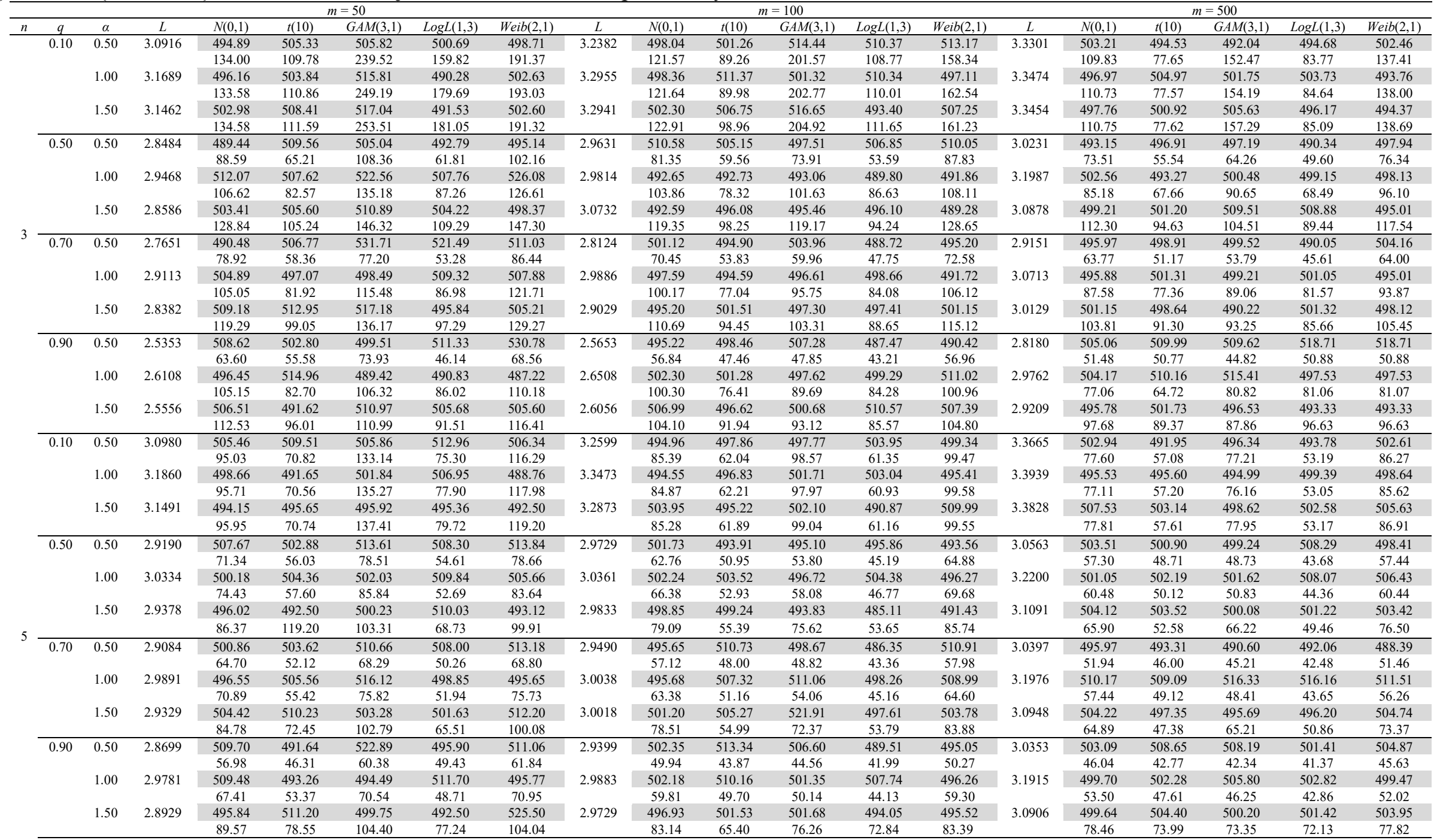

Note: When $\alpha=1$, the proposed W-GWMA $(q, \alpha)$ is equivalent to the W-EWMA( $\lambda$ ) of Li et al. (2010) where $\lambda=1-q$. 
As the Phase II sample size increases, the overall performance of the proposed scheme increases in terms of the $A E Q L$ values. For instance, under the $\operatorname{GAM}(3,1)$ distribution, when $(m, n)=(50,3)$, the proposed W-GWMA $(0.5,0.5)$ scheme yields $A E Q L$ values of 131.4 and 108.36 in Case A and Case E, respectively. However, when $(m, n)=(50,5)$, the proposed W-GWMA $(0.5,0.5)$ scheme yields $A E Q L$ values of 83.63 and 78.51 in Case A and Case E, respectively. From Table 3, in terms of the $A R L$ values, it can be seen that in Case A, when $\alpha=1$, the W-GWMA scheme performs better for large values of $q$ regardless of the size of the shift in the interval $0<q \leq 0.9$. In Case A, when $q$ is between 0.9 and 1 , the sensitivity of the proposed scheme decreases as $q$ increases. However, in Case E, the proposed W-GWMA scheme performs better for large values of $q$ (see Table 4). In terms of the overall performance measures, the W-GWMA $(q, \alpha)$ scheme performs better for large values of $q$ which is equivalent to small values of the smoothing parameter, $\lambda$, of the W-EWMA $(\lambda)$ scheme when $\alpha=1$ with $\lambda=1-q$. Moreover, for both Case A and Case $\mathrm{E}$, the proposed W-GWMA $(q, \alpha)$ scheme performs better under the log-logistic distribution for both small and moderate mean shifts (see Tables 3-5).

\section{Table 3}

Case A OOC characteristics of the run-length distribution and overall performance of the W-GWMA $(q$, $\alpha)($ or W-EWMA $(\lambda))$ scheme when $\alpha=1,(m, n)=(100,5), q=0.5,0.7 \& 0.9$ and $\delta_{\max }=1.5$ for a nominal $A R L_{0}$ value of 500

\begin{tabular}{|c|c|c|c|c|c|c|}
\hline \multirow[b]{2}{*}{ Parameters } & \multirow[b]{2}{*}{ Shifts } & \multicolumn{5}{|c|}{ Distribution } \\
\hline & & $N(\mathbf{0 , 1})$ & $t(10)$ & $G A M(3,1)$ & $\log L(1,3)$ & $\operatorname{Weib}(2,1)$ \\
\hline \multirow{12}{*}{$\begin{array}{c}q=0.5 \\
(\text { i.e. } \lambda=0.5) \\
L=2.9516\end{array}$} & \multirow{2}{*}{0.25} & $141.72(272.67)$ & $66.55(150.32)$ & $167.95(443.52)$ & $54.82(205.02)$ & $179.75(414.42)$ \\
\hline & & $6,22,56,147,549$ & $4,11,27,64,246$ & $5,21,56,149,687$ & $3,8,19,45,188$ & $6,23,64,173,696$ \\
\hline & \multirow{2}{*}{0.50} & $19.43(27.01)$ & $7.82(8.10)$ & $19.97(36.69)$ & $5.03(4.69)$ & $23.57(47.77)$ \\
\hline & & $3,6,11,22,62$ & $2,3,6,9,12$ & $3,5,10,21,66$ & $2,3,4,6,12$ & $3,6,12,24,76$ \\
\hline & \multirow{2}{*}{0.75} & $3.55(1.97)$ & $2.70(0.85)$ & $3.10(1.47)$ & $2.01(0.25)$ & $3.59(2.17)$ \\
\hline & & $2,2,3,4,7$ & $1,2,2,3,4$ & $2,2,3,4,6$ & $2,2,2,2,2$ & $2,2,3,4,7$ \\
\hline & \multirow{2}{*}{1.00} & $2.09(0.65)$ & $1.98(0.56)$ & $2.02(0.34)$ & $1.71(0.46)$ & $2.08(0.50)$ \\
\hline & & $1,2,2,2,3$ & $1,1,2,2,2$ & $2,2,2,2,3$ & $1,1,2,2,2$ & $1,2,2,2,2$ \\
\hline & \multirow{2}{*}{1.50} & $1.63(0.52)$ & $1.50(0.47)$ & $1.74(0.44)$ & $1.25(0.43)$ & $1.73(0.45)$ \\
\hline & & $1,1,2,2,2$ & $1,1,1,2,2$ & $1,1,2,2,2$ & $1,1,1,1,2$ & $1,1,2,2,2$ \\
\hline & $A R A R L$ & 1.64 & 1.29 & 1.51 & 1.00 & 1.56 \\
\hline & PCI & $\begin{array}{l}1.04 \\
1.41\end{array}$ & $\begin{array}{l}1.29 \\
1.08\end{array}$ & $\begin{array}{l}1.01 \\
1.46\end{array}$ & 1.00 & $\begin{array}{l}1.00 \\
1.50\end{array}$ \\
\hline \multirow{12}{*}{$\begin{array}{c}q=0.7 \\
(\text { i.e. } \lambda=0.3) \\
L=2.9950\end{array}$} & \multirow{2}{*}{0.25} & $117.47(249.21)$ & $45.84(147.15)$ & $131.95(458.95)$ & $27.35(99.98)$ & $131.54(345.97)$ \\
\hline & & $6,16,39,101,414$ & $4,9,19,42,149$ & $6,14,35,95,490$ & $4,7,12,23,81$ & $6,16,42,110,525$ \\
\hline & \multirow{2}{*}{0.50} & $13.81(19.13)$ & $6.46(4.81)$ & $12.39(23.83)$ & $4.27(2.09)$ & $14.95(26.85)$ \\
\hline & & $3,6,9,16,32$ & $3,4,5,8,14$ & $3,5,8,13,33$ & $2,3,4,5,8$ & $2,3,5,7,12$ \\
\hline & \multirow{2}{*}{0.75} & $3.60(1.47)$ & $2.61(0.77)$ & $3.19(1.04)$ & $2.11(0.31)$ & $3.55(1.39)$ \\
\hline & & $2,3,3,4,6$ & $2,2,2,3,4$ & $2,3,3,4,5$ & $2,2,2,2,2$ & $2,3,3,4,6$ \\
\hline & \multirow{2}{*}{1.00} & $2.05(0.56)$ & $2.01(0.31)$ & $2.05(0.37)$ & $2.00(0.02)$ & $2.07(0.48)$ \\
\hline & & $2,2,2,3,3$ & $2,2,2,3,3$ & $2,2,2,3,3$ & $2,2,2,3,3$ & $2,2,2,3,3$ \\
\hline & \multirow{2}{*}{1.50} & $1.53(0.18)$ & $1.42(0.13)$ & $1.60(0.04)$ & $1.50(0.00)$ & $1.61(0.08)$ \\
\hline & & $1,1,2,3,3$ & $1,12,3,3$ & $1,1,2,3,3$ & $1,1,1,2,3$ & $1,1,2,2,3$ \\
\hline & $A R A R L$ & 1.93 & 1.21 & 1.95 & 1.00 & 1.97 \\
\hline & PCI & 1.16 & 1.04 & 1.14 & 1.00 & 1.17 \\
\hline \multirow{12}{*}{$\begin{array}{c}q=0.9 \\
(\text { i.e. } \lambda=0.1) \\
L=2.9854\end{array}$} & \multirow{2}{*}{0.25} & $78.81(241.67)$ & $28.64(70.97)$ & $71.93(280.57)$ & $15.75(40.34)$ & $94.28(334.08)$ \\
\hline & & $8,15,27,58,271$ & $6,10,16,27,76$ & $8,13,23,47,218$ & $5,8,11,16,34$ & $8,15,27,59,320$ \\
\hline & \multirow{2}{*}{0.50} & $11.85(8.08)$ & $7.27(3.14)$ & $10.00(7.76)$ & $4.26(1.43)$ & $11.64(9.40)$ \\
\hline & & $5,7,10,14,26$ & $3,4,7,8,13$ & $3,5,8,11,20$ & $2,3,3,5,8$ & $5,7,9,13,24$ \\
\hline & \multirow{2}{*}{0.75} & $3.55(1.27)$ & $3.38(0.77)$ & $3.59(0.89)$ & $3.13(0.33)$ & $4.68(1.14)$ \\
\hline & & $2,3,3,5,7$ & $2,3,4,4,5$ & $3,4,4,5,6$ & $3,3,3,3,4$ & $3,4,4,5,7$ \\
\hline & \multirow[b]{2}{*}{1.00} & $\begin{array}{l}2,5,5,5,7 \\
1.86(0.56)\end{array}$ & $1.75(0.33)$ & $2.00(0.38)$ & $1.58(0.12)$ & $2.31(0.49)$ \\
\hline & & $2,2,3,4,4$ & $2,2,3,3,4$ & $2,2,3,3,4$ & $2,2,3,3,3$ & $2,2,3,4,4$ \\
\hline & \multirow{2}{*}{1.50} & $1.36(0.28)$ & $1.34(0.43)$ & $1.49(0.11)$ & $1.33(0.37)$ & $1.59(0.15)$ \\
\hline & & $1,1,2,2,3$ & $1,1,2,2,3$ & $1,1,2,3,3$ & $1,1,1,2,3$ & $1,1,2,3,3$ \\
\hline & $A R A R L$ & 1.51 & 1.05 & 1.40 & 1.00 & 1.52 \\
\hline & PCI & 1.14 & 1.01 & 1.16 & 1.00 & 1.17 \\
\hline
\end{tabular}

For large shifts in the process location, the performance of the proposed monitoring scheme remains the same regardless of the nature of the underlying distribution. In Case A, the PCI values reveals that when the design parameters $(q, \alpha, L)=(0.5,1,2.9516)$, the monitoring scheme performs $41 \%, 8 \%, 46 \%$ and $50 \%$ better under the $\log L(1,3)$ distribution than the $N(0,1), t(10), \operatorname{GAM}(3,1)$ and $\operatorname{Weib}(2,1)$ distributions, respectively. The W-GWMA $(q, \alpha)$ performs better under the log-logistic distribution followed by the Student's $t$ distribution in terms of the overall performance measures (i.e. ARARL and PCI in Tables 3-5). Next, for Case E, when $\alpha=1$ (see Table 4), the W-GWMA $(q, 1)$ scheme performs better under small and moderate shifts for large values of $q$ regardless of nature of underlying distribution. For large shifts in the location parameter, the performance of the proposed scheme remains the same regardless of the magnitude 
of $q$. Moreover, the proposed W-GWMA $(q, 1)$ scheme performs better under the log-logistic distribution in terms of the $A R L, S D R L, A E Q L, A R A R L$ and $P C I$ values.

\section{Table 4}

Case E OOC characteristics of the run-length distribution and overall performance of the W-GWMA $(q$, $\alpha)$ (or W-EWMA $(\lambda)$ ) scheme when $\alpha=1,(m, n)=(100,5), q=0.5,0.7 \& 0.9$ and $\delta_{\max }=1.5$ for a nominal $A R L_{0}$ value of 500

\begin{tabular}{|c|c|c|c|c|c|c|}
\hline \multirow[b]{2}{*}{ Parameters } & \multirow[b]{2}{*}{ Shifts } & \multicolumn{5}{|c|}{ Distribution } \\
\hline & & $N(0,1)$ & $t(10)$ & $G A M(3,1)$ & $\log L(1,3)$ & Weib $(2,1)$ \\
\hline \multirow{12}{*}{$\begin{array}{c}q=0.5 \\
(\text { i.e. } \lambda=0.5 \text { ) } \\
L=\mathbf{3 . 0 3 6 1}\end{array}$} & \multirow{2}{*}{0.25} & $138.60(261.91)$ & $116.93(193.42)$ & $183.01(555.21)$ & $56.47(307.13)$ & $182.80(409.78)$ \\
\hline & & $5,21,57,142,524$ & $3,12,27,66,248$ & $5,21,57,157,689$ & $3,8,19,45,184$ & $6,23,63,176,728$ \\
\hline & \multirow{2}{*}{0.50} & $18.94(30.28)$ & $13.48(19.38)$ & $20.69(60.98)$ & $4.92(5.10)$ & $23.14(47.43)$ \\
\hline & & $2,5,11,22,59$ & $2,3,5,9,21$ & $2,5,10,21,65$ & $2,3,4,6,12$ & $3,6,12,25,76$ \\
\hline & \multirow{2}{*}{0.75} & $6.25(5.53)$ & $2.07(1.01)$ & $2.96(1.60)$ & $1.64(0.53)$ & $3.65(2.07)$ \\
\hline & & $2,3,5,8,16$ & $1,1,2,3,4$ & $1,2,3,3,6$ & $1,1,2,2,2$ & $1,2,3,4,7$ \\
\hline & \multirow{2}{*}{1.00} & $3.33(2.07)$ & $1.13(0.53)$ & $1.65(0.56)$ & $1.07(0.25)$ & $1.77(0.65)$ \\
\hline & & $1,2,3,4,7$ & $1,1,1,2,2$ & $1,1,2,2,2$ & $1,1,1,1,2$ & $1,1,2,2,3$ \\
\hline & \multirow{2}{*}{1.50} & $1.27(0.46)$ & $1.12(0.34)$ & $1.14(0.35)$ & $1.00(0.00)$ & $1.22(0.42)$ \\
\hline & & $1,1,1,2,2$ & $1,1,1,1,2$ & $1,1,1,1,2$ & $1,1,1,1,1$ & $1,1,1,1,2$ \\
\hline & $A R A R L$ & 1.93 & 1.20 & 1.90 & 1.00 & 1.95 \\
\hline & PCI & 1.42 & 1.13 & 1.24 & 1.00 & 1.49 \\
\hline \multirow{12}{*}{$\begin{array}{c}q=0.7 \\
(\text { i.e. } \lambda=0.3 \text { ) } \\
L=\mathbf{3 . 0 0 2 8}\end{array}$} & \multirow{2}{*}{0.25} & $115.30(300.40)$ & $82.25(93.25)$ & $123.05(404.93)$ & $26.77(107.07)$ & $138.36(412.01)$ \\
\hline & & $5,15,38,103,444$ & $4,9,18,40,146$ & $5,14,34,92,448$ & $3,6,11,23,80$ & $5,16,41,113,548$ \\
\hline & \multirow{2}{*}{0.50} & $13.10(15.55)$ & $9.03(4.65)$ & $11.69(17.37)$ & $3.83(2.19)$ & $14.61(37.95)$ \\
\hline & & $2,5,9,15,37$ & $2,3,5,7,14$ & $3,5,7,13,34$ & $2,2,2,2,2$ & $3,5,9,16,42$ \\
\hline & \multirow{2}{*}{0.75} & $3.11(1.60)$ & $2.06(0.88)$ & $2.73(1.10)$ & $1.70(0.49)$ & $3.08(1.46)$ \\
\hline & & $1,2,3,4,6$ & $1,1,2,2,4$ & $1,2,3,3,5$ & $1,1,2,2,2$ & $1,2,3,4,6$ \\
\hline & \multirow{2}{*}{1.00} & $1.78(0.70)$ & $1.36(0.54)$ & $1.70(0.52)$ & $1.10(0.30)$ & $1.80(0.60)$ \\
\hline & & $1,1,2,2,3$ & $1,1,1,2,2$ & $1,1,2,2,2$ & $1,1,1,1,2$ & $1,1,2,2,3$ \\
\hline & \multirow{2}{*}{1.50} & $1.30(0.47)$ & $1.14(0.35)$ & $1.20(0.40)$ & $1.00(0.06)$ & $1.27(0.44)$ \\
\hline & & $1,1,1,2,2$ & $1,1,1,1,2$ & $1,1,1,1,2$ & $1,1,1,1,1$ & $1,1,1,2,2$ \\
\hline & ARARL & 1.89 & 1.20 & 1.51 & 1.00 & 1.90 \\
\hline & PCI & 1.41 & 1.13 & 1.20 & 1.00 & 1.43 \\
\hline \multirow{12}{*}{$\begin{array}{c}q=0.9 \\
(\text { i.e. } \lambda=0.1) \\
L=\mathbf{2 . 9 8 8 3}\end{array}$} & \multirow{2}{*}{0.25} & $79.63(253.14)$ & $52.71(58.96)$ & $72.74(315.13)$ & $13.32(26.31)$ & $85.81(263.33)$ \\
\hline & & $5,13,25,58,291$ & $3,8,14,25,77$ & $5,11,21,44,240$ & $3,6,9,14,33$ & $5,13,25,58,317$ \\
\hline & \multirow{2}{*}{0.50} & $9.75(9.13)$ & $7.24(3.27)$ & $8.17(7.13)$ & $3.48(1.59)$ & $9.92(10.24)$ \\
\hline & & $2,5,8,12,24$ & $2,3,4,7,11$ & $2,4,7,10,19$ & $2,2,3,4,6$ & $3,5,8,12,24$ \\
\hline & \multirow{2}{*}{0.75} & $2.97(1.42)$ & $2.03(0.86)$ & $2.60(0.96)$ & $1.68(0.49)$ & $2.92(1.26)$ \\
\hline & & $1,2,3,4,6$ & $1,1,2,2,4$ & $1,2,2,3,4$ & $1,1,2,2,2$ & $1,2,3,4,5$ \\
\hline & \multirow{2}{*}{1.00} & $1.75(0.69)$ & $1.34(0.52)$ & $1.68(0.51)$ & $1.09(0.28)$ & $1.77(0.57)$ \\
\hline & & $1,1,2,3,3$ & $1,1,1,2,2$ & $1,1,2,2,2$ & $1,1,1,1,2$ & $1,1,2,2,3$ \\
\hline & \multirow{2}{*}{1.50} & $1.29(0.46)$ & $1.13(0.35)$ & $1.18(0.38)$ & $1.00(0.04)$ & $1.26(0.44)$ \\
\hline & & $1,1,1,2,2$ & $1,1,1,1,2$ & $1,1,1,1,2$ & $1,1,1,1,1$ & $1,1,1,2,2$ \\
\hline & $A R A R L$ & 1.71 & 1.19 & 1.60 & 1.00 & 1.69 \\
\hline & PCI & 1.36 & 1.13 & 1.14 & 1.00 & 1.34 \\
\hline
\end{tabular}

From Table 5, the following can be observed:

- When $q$ is kept fixed, the sensitivity of the W-GWMA $(q, \alpha)$ scheme increases for small values of $\alpha$. However, for large value of $\alpha$, its sensitivity decreases.

- When the value of $\alpha$ is kept fixed, the variability in the $A R L$ values decreases as the values of $q$ increases and consequently, the sensitivity of the proposed W-GWMA scheme increases.

- The proposed W-GWMA performs better under the log-logistic distribution.

\subsection{Performance comparison}

In this section, the proposed W-GWMA $(q, \alpha)$ scheme is compared to several monitoring schemes including the traditional Shewhart $\bar{X}$, W-Shewhart, $\bar{X}$-CUSUM, $\bar{X}$-EWMA, median CUSUM (denoted as $\tilde{X}$ CUSUM), median EWMA (denoted as $\tilde{X}$-EWMA), W-CUSUM, W-EWMA and various GWMA schemes under the $N(0,1)$ and log-logistic distributions. For a fair comparison, the performance of competing schemes are investigated for $(m, n)=(100,5)$ in terms of the $A R L$ and $A E Q L$ values for a nominal $A R L_{0}$ value of 500 with and $\delta_{\max }=2$. The GWMA and EWMA-type monitoring schemes are investigated in Case $\mathrm{E}$ when $q=0.9$ (which is equivalent to the smoothing parameter, $\lambda=0.1$ ) and $\alpha=0.5$ and 1 . In Table 6, the proposed monitoring scheme is compared to different schemes in terms of the $A R L$ and $A E Q L$ values. The scheme that performs best is shaded in grey. It can be seen that when $\alpha=1$, the proposed WGWMA scheme is equivalent to the W-EWMA scheme of Li et al. (2010), i.e. they yield the same exact OOC performance as shown in Table 6 (Columns 10 and 14). Under the $N(0,1)$ distribution, $\bar{X}$-GWMA $(0.9,1)$ and $\bar{X}$-EWMA $(0.1)$ schemes outperform the competing schemes as they yield the lowest $A E Q L$ as compared to other competing schemes. 
Table 5

Case E OOC characteristics of the run-length distribution and overall performance of the W-GWMA scheme when $(m, n)=(100,5), \alpha=0.5,1.5 \& 2.5, q=0.5 \& 0.9$ and $\delta_{\max }=1.5$ for a nominal $A R L_{0}$ value of 500

\begin{tabular}{|c|c|c|c|c|c|c|}
\hline \multirow[b]{2}{*}{ Parameters } & \multirow[b]{2}{*}{ Shifts } & \multicolumn{5}{|c|}{ Distribution } \\
\hline & & $N(0,1)$ & $t(10)$ & $G A M(3,1)$ & $\log L(1,3)$ & $\operatorname{Weib}(2,1)$ \\
\hline \multirow{12}{*}{$\begin{array}{c}q=\mathbf{0 . 5} \\
\alpha=\mathbf{0 . 5} \\
L=\mathbf{2 . 9 2 2 9}\end{array}$} & \multirow{2}{*}{0.25} & $121.55(252.32)$ & $57.47(146.67)$ & $157.60(542.07)$ & $42.63(193.42)$ & $155.29(386.29)$ \\
\hline & & $6,20,47,116,467$ & $4,12,24,54,186$ & $6,20,46,124,570$ & $4,9,18,37,123$ & $6,21,52,131,610$ \\
\hline & \multirow{2}{*}{0.50} & $17.41(22.48)$ & $7.83(6.65)$ & $18.17(55.19)$ & $5.62(4.16)$ & $20.07(30.65)$ \\
\hline & & $3,6,11,21,50$ & $2,4,6,10,19$ & $3,6,11,20,5$ & $2,3,5,7,13$ & $3,7,12,23,59$ \\
\hline & \multirow{2}{*}{0.75} & $3.68(2.29)$ & $2.14(1.18)$ & $3.47(1.98)$ & $1.65(0.65)$ & $3.86(2.33)$ \\
\hline & & $1,2,3,5,8$ & $1,1,2,3,4$ & $1,2,3,4,7$ & $1,1,1,1,1$ & $1,2,3,5,8$ \\
\hline & \multirow[b]{2}{*}{1.00} & $\begin{array}{l}1,2,3,5,8 \\
1.84(0.90)\end{array}$ & $\begin{array}{l}1,1,2,5,4 \\
1.13(0.57)\end{array}$ & $\begin{array}{l}1,2,5,4,1 \\
1.70(0.70)\end{array}$ & $\begin{array}{l}1,1,1,1,1 \\
1.04(0.20)\end{array}$ & $1.84(0.82)$ \\
\hline & & $1,1,2,2,4$ & $1,1,1,2,2$ & $1,1,2,2,3$ & $1,1,1,1,1$ & $1,1,2,2,3$ \\
\hline & \multirow[b]{2}{*}{1.50} & $1.25(0.47)$ & $1.12(0.34)$ & $1.12(0.33)$ & $1.00(0.02)$ & $1.18(0.40)$ \\
\hline & & $1,1,1,1,2$ & $1,1,1,1,2$ & $1,1,1,1,2$ & $1,1,1,1,1$ & $1,1,1,1,2$ \\
\hline & ARARL & 1.87 & 1.04 & 1.96 & $\mathbf{1 . 0 0}$ & 2.08 \\
\hline & $P C I$ & 1.39 & 1.13 & 1.19 & 1.00 & 1.44 \\
\hline \multirow{12}{*}{$\begin{array}{c}q=0.5 \\
\alpha=1.5 \\
L=2.9433\end{array}$} & \multirow{2}{*}{0.25} & $137.81(279.99)$ & $74.68(161.48)$ & $193.41(462.54)$ & $46.94(154.64)$ & $201.21(452)$. \\
\hline & & $5,25,67,174,589$ & $4,13,33,81,299$ & $5,25,71,193,730$ & $2,6,14,38,166$ & $6,29,82,208,814$ \\
\hline & \multirow{2}{*}{0.50} & $22.30(33.65)$ & $8.76(11.72)$ & $24.21(41.33)$ & $6.01(4.55)$ & $27.71(43.23)$ \\
\hline & & $2,6,13,27,82$ & $2,3,6,10,26$ & $2,6,13,27,92$ & $2,2,3,4,9$ & $2,7,15,33,106$ \\
\hline & \multirow{2}{*}{0.75} & $5.72(4.64)$ & $3.11(2.02)$ & $5.41(4.78)$ & $1.97(0.58)$ & $6.63(6.93)$ \\
\hline & & $2,3,4,6,15$ & $1,2,3,4,7$ & $2,3,4,5,12$ & $1,2,2,2,3$ & $2,3,4,8,20$ \\
\hline & \multirow{2}{*}{1.00} & $2.89(1.98)$ & $2.02(1.02)$ & $2.59(1.89)$ & $1.54(0.41)$ & $3.03(2.01)$ \\
\hline & & $1,1,2,4,5$ & $1,1,2,2,3$ & $1,2,2,3,4$ & $1,1,2,2,2$ & $1,2,3,4,6$ \\
\hline & \multirow{2}{*}{1.50} & $1.91(0.83)$ & $1.37(0.40)$ & $1.64(0.53)$ & $1.25(0.34)$ & $1.98(0.96)$ \\
\hline & & $1,1,2,2,3$ & $1,1,1,2,2$ & $1,1,2,2,2$ & $1,1,1,2,2$ & $1,1,2,2,2$ \\
\hline & $A R A R L$ & 1.92 & 1.72 & 1.87 & $\mathbf{1 . 0 0}$ & 1.95 \\
\hline & PCI & 1.45 & 1.12 & $\begin{array}{l}1.01 \\
1.32\end{array}$ & 1.00 & 1.53 \\
\hline \multirow{12}{*}{$\begin{array}{c}q=0.9 \\
\alpha=1.5 \\
L=2.9121\end{array}$} & \multirow{2}{*}{0.25} & $103.22(270.66)$ & $37.6(131.26)$ & $111.93(389.33)$ & $22.93(127.16)$ & $119.14(343.93)$ \\
\hline & & $5,14,32,87,404$ & $3,8,16,34,119$ & $5,12,27,75,396$ & $3,6,9,17,58$ & $5,14,33,42,482$ \\
\hline & \multirow{2}{*}{0.50} & $11.77(15.44)$ & $5.55(4.20)$ & $9.84(20.74)$ & $3.47(1.60)$ & $12.21(18.66)$ \\
\hline & & $2,5,8,13,33$ & $2,3,5,7,12$ & $2,4,7,11,25$ & $2,2,3,4,6$ & $2,5,8,13,33$ \\
\hline & \multirow{2}{*}{0.75} & $3.00(1.48)$ & $2.00(0.92)$ & $2.59(0.99)$ & $1.58(0.51)$ & $2.90(1.28)$ \\
\hline & & $1,2,3,4,6$ & $1,1,2,2,4$ & $1,2,2,3,4$ & $1,1,2,2,2$ & $1,2,3,4,5$ \\
\hline & \multirow{2}{*}{1.00} & $1.73(0.72)$ & $1.32(0.53)$ & $1.61(0.53)$ & $1.05(0.22)$ & $1.71(0.60)$ \\
\hline & & $1,1,2,2,3$ & $1,1,1,2,2$ & $1,1,2,2,2$ & $1,1,1,1,1$ & $1,1,2,2,3$ \\
\hline & \multirow{2}{*}{1.50} & $1.25(0.45)$ & $1.12(0.33)$ & $1.13(0.33)$ & $1.00(0.03)$ & $1.20(0.40)$ \\
\hline & & $1,1,1,1,2$ & $1,1,1,1,2$ & $1,1,1,1,2$ & $1,1,1,1,1$ & $1,1,1,1,2$ \\
\hline & $A R A R L$ & 1.89 & 1.21 & 1.67 & 1.00 & 1.91 \\
\hline & PCI & 1.12 & 1.03 & 1.04 & 1.00 & 1.13 \\
\hline & 025 & $144.44(289.13)$ & $65.87(155.95)$ & $175.87(440.05)$ & $47.46(134.34)$ & $181.22(397.51)$ \\
\hline & 0.25 & $6,21,58,147,563$ & $4,11,27,64,240$ & $5,21,59,159,709$ & $3,8,17,42,172$ & $6,24,65,176,726$ \\
\hline & 0.50 & $20.08(32.13)$ & $7.66(7.58)$ & $19.55(35.32)$ & $4.59(3.86)$ & $23.04(37.25)$ \\
\hline & 0.50 & $3,6,11,23,65$ & $2,4,5,9,21$ & $3,5,10,20,65$ & $2,3,4,5,10$ & $3,6,12,25,78$ \\
\hline & & $3.53(2.01)$ & $2.27(1.11)$ & $3.07(1.52)$ & $1.70(0.61)$ & $3.48(1.95)$ \\
\hline$q=0.9$ & 0.75 & $1,2,3,4,7$ & $1,1,2,3,4$ & $1,2,3,4,5$ & $1,1,2,2,3$ & $1,2,3,4,7$ \\
\hline$\alpha=2.5$ & & $1.91(0.89)$ & $1.40(0.68)$ & $1.73(0.67)$ & $1.06(0.24)$ & $1.90(0.77)$ \\
\hline$L=2.9453$ & 1.00 & $1,1,2,3,3$ & $1,1,1,2,3$ & $1,1,2,2,3$ & $1,1,1,1,2$ & $1,1,2,2,3$ \\
\hline & & $1.30(0.54)$ & $1.14(0.40)$ & $1.15(0.37)$ & $1.00(0.03)$ & $1.23(0.44)$ \\
\hline & 1.50 & $1,1,1,2,2$ & $1,1,1,1,2$ & $1,1,1,1,2$ & $1,1,1,1,1$ & $1,1,1,1,2$ \\
\hline & $A R A R L$ & 1.69 & 1.21 & 1.60 & 1.00 & 1.70 \\
\hline & PCI & 1.13 & 1.03 & 1.05 & 1.00 & 1.13 \\
\hline
\end{tabular}

This was expected since parametric monitoring schemes perform better than their nonparametric counterparts when the assumption of normality is satisfied. Table 6 shows that under non-normal distributions, the proposed W-GWMA $(0.9,1)$ outperforms the $\tilde{X}$-GWMA and $\bar{X}$-GWMA as well as all other competing schemes considered in this paper (see Table 6). Note that the proposed W-GWMA scheme is more flexible than the W-EWMA scheme because of the extra design parameter, $\alpha$, which can be set according to the operator's expectations. It can be observed that when $q$ is kept fixed; the W-GWMA scheme performs better than the W-EWMA scheme for $\alpha<1$.

\section{Illustrative example}

To illustrate the implementation and application of the proposed W-GWMA monitoring scheme, two sets of data on the inside diameters of piston rings manufactured by a forging process are used (Montgomery, 2005, page 223). The first set of data contains twenty-five Phase I samples, each of size $n=5(m=125)$ collected when the process was considered to be IC. These data are used as the Phase I data for which a goodness of fit test for normality is not rejected. The second set of data contains fifteen test samples each of size $n=5$ which are considered to be the Phase II data. 
Table 6

$\underline{\text { Performance comparison of different monitoring schemes when }(m, n)=(100,5), q=0.9, \alpha=0.5 \& 1 \text { and } \delta_{\max }=2 \text { for a nominal } A R L_{0} \text { of } 500}$

\begin{tabular}{|c|c|c|c|c|c|c|c|c|c|c|c|c|c|c|}
\hline $\begin{array}{c}\text { Distributio } \\
\text { n }\end{array}$ & Shift & $\begin{array}{c}\bar{X} \\
\text { scheme }\end{array}$ & $\begin{array}{c}\text { W- } \\
\text { Shewhart }\end{array}$ & $\begin{array}{c}\bar{X}- \\
\text { CUSUM }\end{array}$ & $\begin{array}{c}\bar{X} \text {-EWMA } \\
(0.1)\end{array}$ & $\begin{array}{c}\widetilde{X}- \\
\text { CUSUM }\end{array}$ & $\begin{array}{c}\widetilde{X}- \\
\mathbf{E W M} \\
\mathbf{A}(0.1)\end{array}$ & $\underset{\mathbf{M}}{\mathbf{W}-}$ & $\begin{array}{c}\text { W-EWMA } \\
(\mathbf{0 . 1 )}\end{array}$ & $\begin{array}{c}\bar{X} \text {-GWMA }(0.9, \\
\text { 1) }\end{array}$ & $\begin{array}{c}\widetilde{X} \text {-GWMA } \\
\text { 1) }\end{array}$ & $\begin{array}{c}\widetilde{X} \text {-GWMA } \\
0.5)\end{array}$ & $\begin{array}{c}\text { W- } \\
\text { GWM } \\
\text { A (0.9, } \\
1)\end{array}$ & $\begin{array}{c}\text { W- } \\
\text { GWM } \\
\text { A (0.9, } \\
0.5)\end{array}$ \\
\hline \multirow{7}{*}{$N(\mathbf{0}, \mathbf{1})$} & 0.00 & 500.36 & 501.76 & 506.12 & 502.32 & 513.39 & 511.52 & 503.36 & 499.83 & 502.32 & 511.52 & 509.09 & 499.83 & 501.00 \\
\hline & 0.25 & 170.26 & 186.45 & 78.19 & 76.24 & 92.92 & 91.02 & 87.52 & 79.63 & 76.24 & 91.02 & 93.05 & 79.63 & 74.32 \\
\hline & 0.50 & 63.4 & 70.06 & 21.49 & 11.04 & 27.82 & 27.09 & 24.31 & 9.75 & 11.04 & 27.09 & 28.41 & 9.75 & 12.17 \\
\hline & 1.00 & 7.38 & 9.81 & 4.44 & 2.81 & 5.33 & 5.29 & 4.82 & 2.97 & 2.81 & 5.29 & 5.32 & 2.97 & 3.68 \\
\hline & 1.50 & 2.94 & 3.24 & 2.06 & 1.98 & 3.30 & 3.31 & 3.02 & 1.75 & 1.98 & 3.31 & 3.36 & 1.75 & 2.04 \\
\hline & 2.00 & 1.82 & 2.66 & 1.52 & 1.31 & 3.01 & 3.02 & 3.12 & 1.29 & 1.31 & 3.02 & 3.03 & 1.29 & 1.41 \\
\hline & $A E Q L$ & 109.49 & 122.17 & 71.34 & 65.04 & 91.33 & 90.23 & 88.54 & 66.86 & 65.04 & 89.28 & 90.11 & 66.86 & 67.71 \\
\hline \multirow{7}{*}{$\operatorname{GAM}(1,3)$} & 0.00 & 540.56 & 498.1 & 523.39 & 531.54 & 520.11 & 509.63 & 501.79 & 500.44 & 531.54 & 509.63 & 512.43 & 500.44 & 494.29 \\
\hline & 0.25 & 192.20 & 139.33 & 121.07 & 103.03 & 75.14 & 74.84 & 78.46 & 72.74 & 103.03 & 74.84 & 76.23 & 72.74 & 76.50 \\
\hline & 0.50 & 70.36 & 61.04 & 17.48 & 14.20 & 12.05 & 11.95 & 9.97 & 8.17 & 14.20 & 11.95 & 12.04 & 8.17 & 11.07 \\
\hline & 1.00 & 13.01 & 9.18 & 8.41 & 4.48 & 3.31 & 3.36 & 3.26 & 2.60 & 4.48 & 3.36 & 3.37 & 2.60 & 3.37 \\
\hline & 1.50 & 5.72 & 4.28 & 3.98 & 3.33 & 2.76 & 2.83 & 2.63 & 1.68 & 3.33 & 2.83 & 2.89 & 1.68 & 1.95 \\
\hline & 2.00 & 4.00 & 3.01 & 2.53 & 2.59 & 1.93 & 1.90 & 1.70 & 1.18 & 2.59 & 1.90 & 2.01 & 1.18 & 1.35 \\
\hline & $A E Q L$ & 146.22 & 115.30 & 97.85 & 82.37 & 73.04 & 71.78 & 70.14 & 64.36 & 82.37 & 71.78 & 72.11 & 64.36 & 67.40 \\
\hline \multirow{7}{*}{$\log L(1,3)$} & 0.00 & 536.41 & 498.1 & 541.05 & 519.83 & 508.87 & 510.21 & 502.34 & 510.59 & 519.83 & 510.21 & 507.35 & 510.59 & 503.77 \\
\hline & 0.25 & 176.72 & 140.71 & 37.23 & 36.34 & 21.33 & 20.48 & 18.19 & 13.32 & 36.34 & 20.48 & 22.01 & 13.32 & 16.92 \\
\hline & 0.50 & 66.21 & 59.14 & 13.63 & 11.33 & 7.32 & 6.58 & 5.14 & 3.48 & 11.33 & 6.58 & 6.66 & 3.48 & 4.79 \\
\hline & 1.00 & 11.2 & 8.34 & 8.42 & 7.88 & 2.10 & 2.09 & 1.99 & 1.68 & 7.88 & 2.09 & 2.12 & 1.68 & 1.93 \\
\hline & 1.50 & 4.84 & 3.98 & 4.29 & 4.91 & 2.05 & 2.07 & 2.04 & 1.09 & 4.91 & 2.07 & 2.05 & 1.09 & 1.24 \\
\hline & 2.00 & 3.13 & 2.68 & 2.43 & 2.34 & 1.22 & 1.20 & 1.19 & 1.00 & 2.34 & 1.20 & 1.31 & 1.00 & 1.02 \\
\hline & $A E Q L$ & 131.16 & 113.49 & 85.03 & 84.42 & 69.23 & 68.09 & 67.18 & 63.53 & 84.42 & 68.09 & 69.01 & 63.53 & 64.17 \\
\hline
\end{tabular}


In Case A, when $\alpha=1$ and $q=0.9$, the proposed W-GWMA scheme is equivalent to the W-EWMA of Li et al. (2010) with $\lambda=0.1$. For a nominal $A R L_{0}$ value of 500, we found $L=3.2123$ so that the asymptotic control limits $\left(L C L_{A}, U C L_{A}\right)=(187.28,467.72)$ that yield an attained $A R L_{0}$ value of 500.64 (obtained using SAS 9.4). In Case E, we found $L=2.9402$ so that the W-GWMA $(0.9,1)$ and W-EWMA $(0.1)$ yield an attained $A R L_{0}$ value of 501.26. A plot of the Case A and Case E charting statistics of the proposed W-GWMA is shown in Fig. 1(a). It can be seen that in Case A, the W-GWMA $(0.9,1)$ scheme chart signals on the $13^{\text {th }}$ sample in the prospective phase. However, in Case E, W-GWMA $(0.9,1)$ gives a signal on the $12^{\text {th }}$ sample in the prospective phase. When $\alpha \neq 1$, the control limits coefficients of the W-GWMA $(0.9,0.5)$ and W-GWMA $(0.9,1.5)$ schemes are found to be equal to 3.1302 and 2.9761, respectively, so that they yield the attained $A R L_{0}$ values of 500.32 and 502.51, respectively. A plot of the charting statistics of the W-GWMA $(0.9,0.5)$ and W-GWMA $(0.9,1.5)$ are shown in Fig. 1(b). It can be seen that both schemes signal on the $12^{\text {th }}$ sample in the prospective phase.

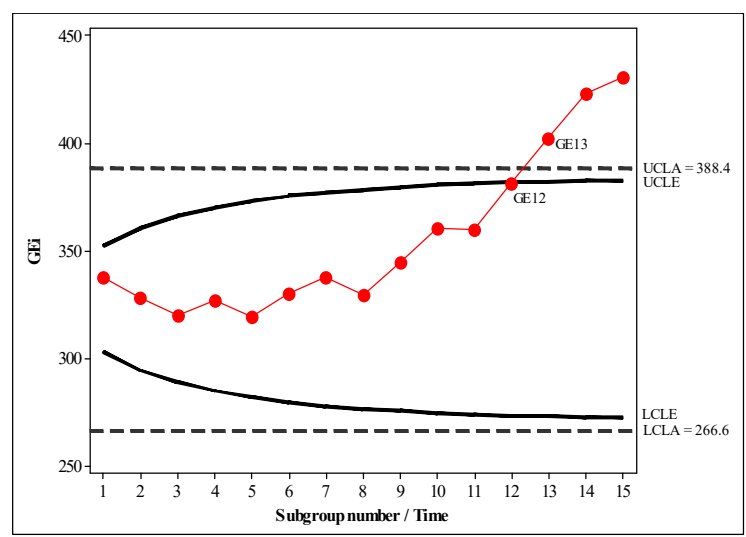

(a) W-EWMA $\equiv \mathrm{W}$-GWMA control schemes $(q=0.9$, $\alpha=1$ and $\lambda=0.1)$

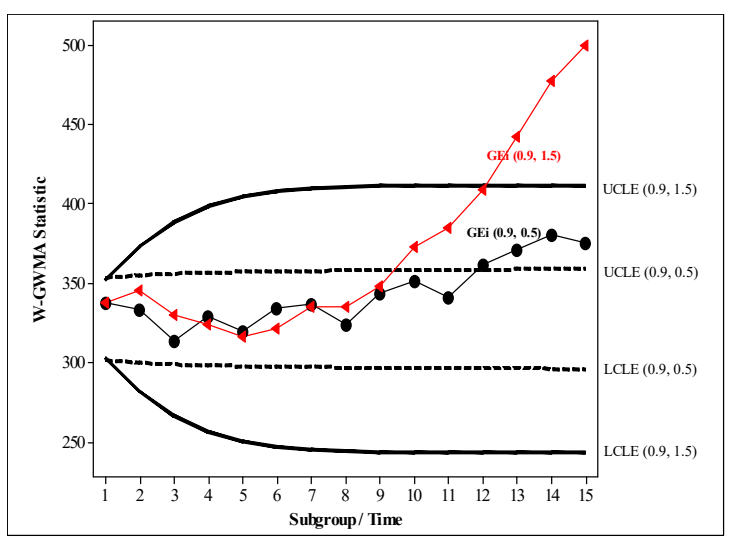

(b) W-GWMA control schemes $(q=0.9, \alpha=0.5$ and 1.5)

Fig. 1. Proposed monitoring schemes for the Montgomery (2005)'s piston ring data

\section{Effect of the design parameters and Phase I sample size on the IC and OOC performances of the proposed monitoring scheme}

In this section, the effect of the design parameters (i.e. $q, \alpha$ and $L$ ) and the Phase I sample size $m$ on the Phase II performance of the proposed monitoring scheme is investigated in Case E.

\subsection{Effect of the design parameters on the Phase II performance of the W-GWMA scheme}

More often, the design of nonparametric charts requires tables for the optimal parameters. When these tables are not available, one would need to know the relationship between the parameters and the attained $A R L_{0}$ in order to estimate as quick as possible the optimal value to be used. This section investigates the relationship between the design parameters and the $A R L_{0}$ and $A E Q L$ metrics.

Fig. 2 displays the attained $A R L_{0}$ values for different combinations of $q$ and $\alpha$ when $(m, n)=(100,5)$ and $L=2,2.5,2.75$ and 3. Comparing the graphs in Fig. 2, it can be observed that the attained $A R L_{0}$ value is proportional to the distance between the control limits from the centerline (i.e. $L$ ), that is, as $L$ increases (decreases), the attained $A R L_{0}$ values increases (decreases). In Fig. 2 (a), it can be seen that the $A R L_{0}$ is an increasing function of $q$. When $L \in(2,2.5)$, the larger the value of $\alpha$, the higher the attained $A R L_{0}$ (see Figs. 2 (a) and (b)). Figs. 2 (b) and (c) show that the attained $A R L_{0}$ is a decreasing function of $q$ in the interval $(0.5,0.8)$ when $L=2.5$ and 2.75 for $\alpha=0.5,0.75$ and 1 , which makes it difficult to reach the high desired value of $\tau$. However, the attained $A R L_{0}$ is an increasing function of $q$ in the interval $(0.8,1)$. In Fig. 2 (d), the attained $A R L_{0}$ is a decreasing function of $q$ when $q \leq 0.8$ for $\alpha \geq 0.75$.

In Fig. 3, it can be easily observed that the overall performance $A E Q L$ is a decreasing function of $q$. The larger the value of $q$, the more efficient the $\mathrm{W}-\operatorname{GWMA}(q, \alpha)$ scheme (see Figs. 3 (a) and (b)). In Fig. 3 (b), 
it can be observed that the $A E Q L$ is a decreasing function of $\alpha$ in the interval $(0.25,1)$ and $A E Q L$ is an increasing function of $\alpha$ when $\alpha>1$.

Therefore, based on Fig. 3, it is apparent that the W-GWMA $(q, \alpha)$ is more efficient for large value of $q$ and small values of $\alpha$.

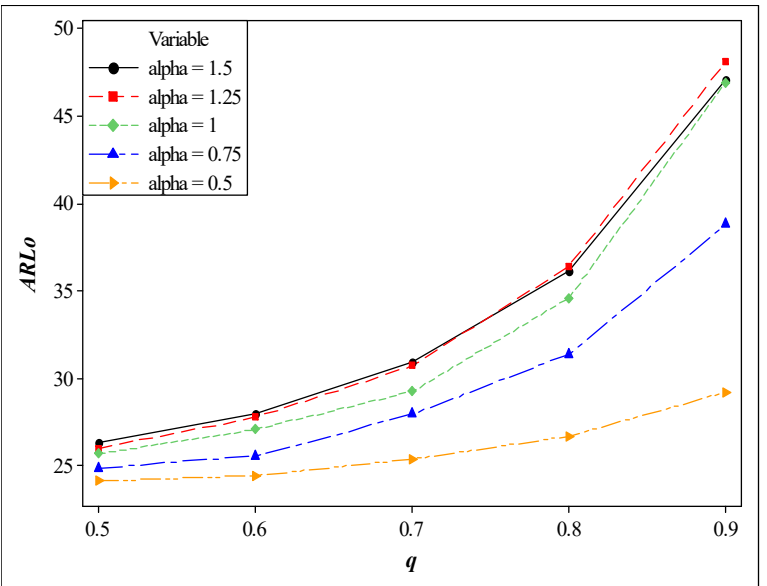

(a) $L=2$

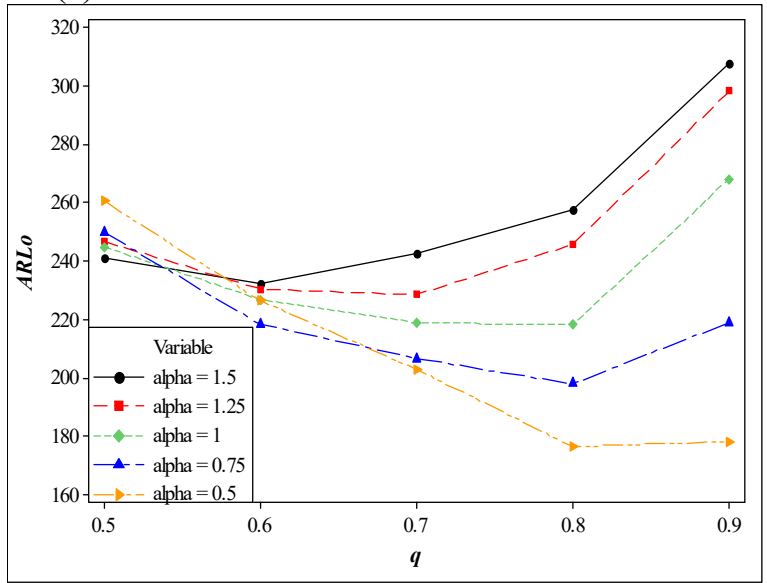

(c) $L=2.75$

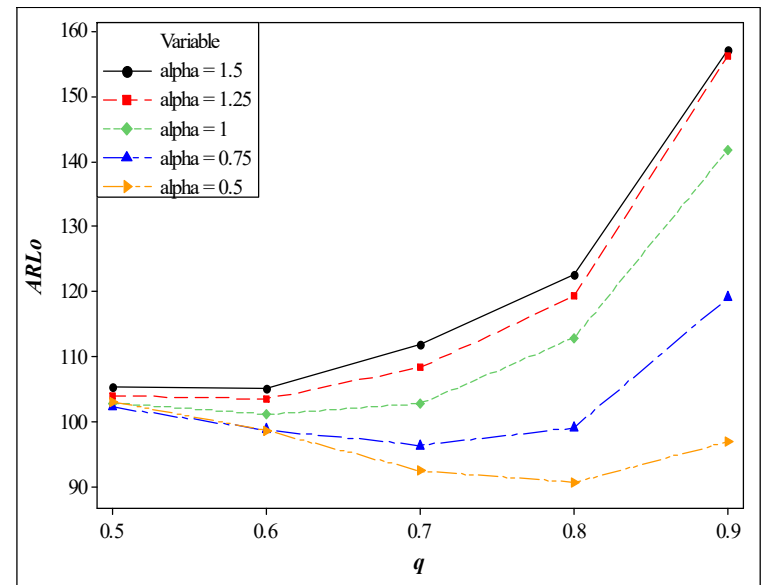

(b) $L=2.5$

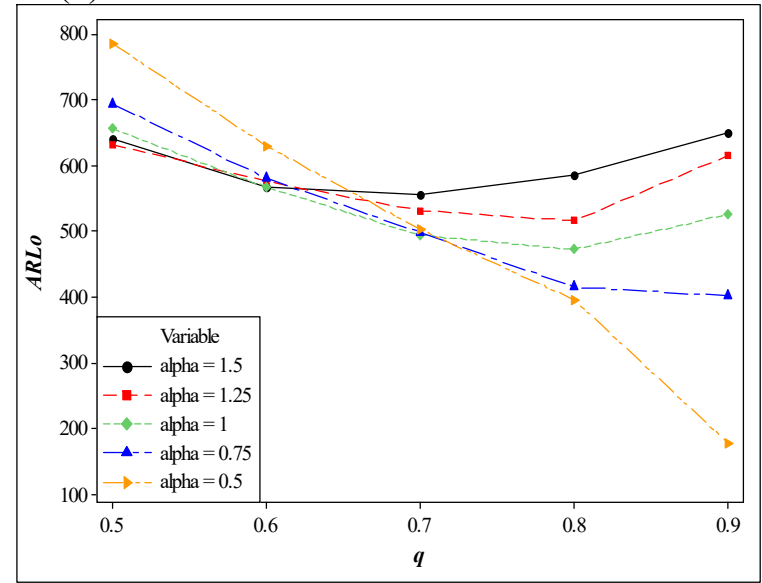

(d) $L=3.00$

Fig. 2. Attained $A R L_{0}$ values versus $q$ values for different $\alpha$ and $L$ values when $(m, n)=(100,5)$

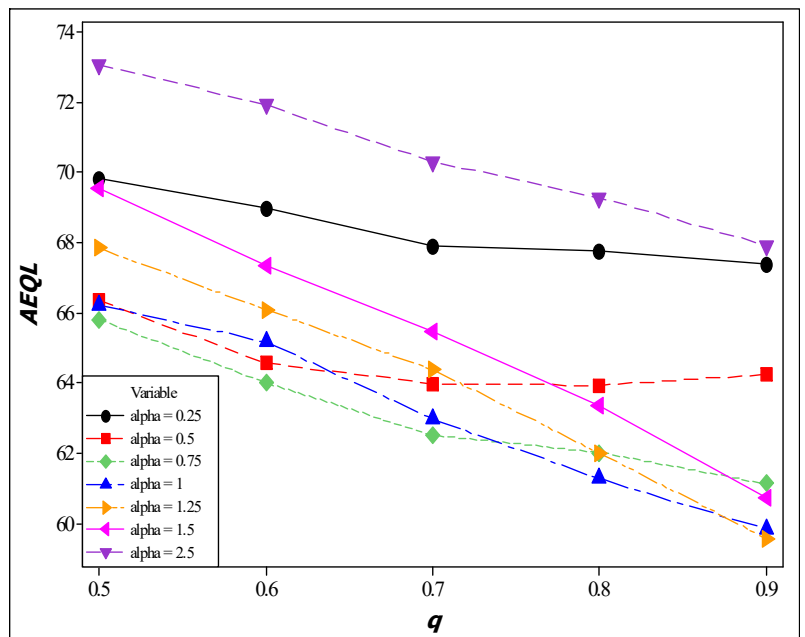

(a) $A E Q L$ versus $q$

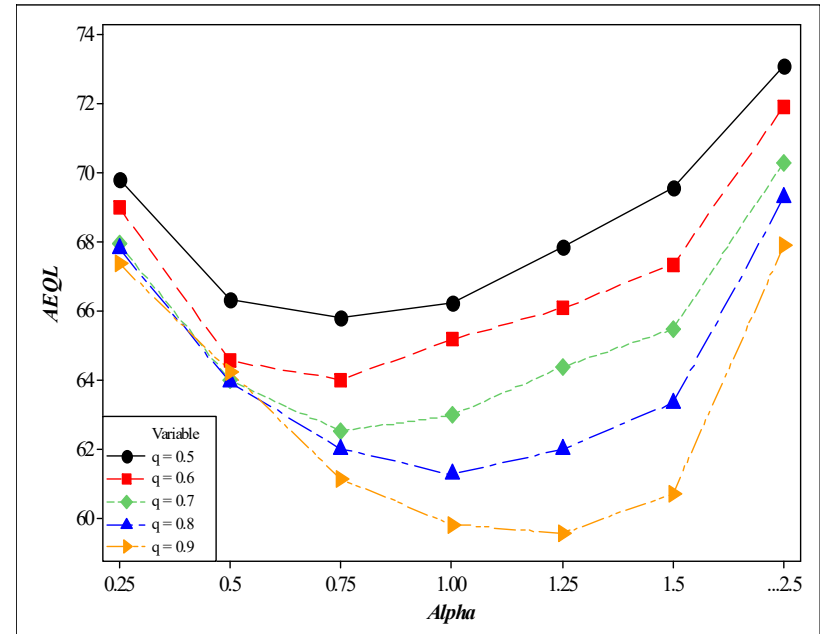

(b) $A E Q L$ versus $\alpha$

Fig. 3. Overall performance measure of the W-GWMA $(q, \alpha)$ scheme when $(m, n)=(100,5)$ 


\subsection{Effect of the Phase I sample size on the Phase II performance of the W-GWMA}

Parameter estimation deteriorates considerably the performance of control schemes (Jensen et al., 2006; Zhang et al., 2014). It is also well-known that a control scheme with known process parameters (Case K) will perform better than the same control scheme with unknown process parameters (Case U). Therefore, it is very important to know the minimum number of the IC Phase I observations, i.e. $m_{\text {opt }}$, that allows a control scheme to perform in Case $U$ as if the process parameters were known. In this section, the effect of the Phase I sample size is investigated in order to know the minimum Phase I sample size that guarantees stability in the performance of the W-GWMA control scheme. Thus, the impact (or effect) of the parameter estimation (from the Phase I sample) on the Phase II performance of the W-GWMA scheme is investigated in respect of the IC characteristics of the conditional run-length distribution, i.e. the mean and standard deviation of the IC conditional average run-length $\left(C A R L_{0}\right)$ distribution denoted $C A A R L_{0}$ and $C S D A R L_{0}$, respectively. Therefore, $m_{\text {opt }}$ is the minimum Phase I sample size such that the $C A A R L_{0}$ value is near the nominal $A R L_{0}$ and the $C S D A R L_{0}$ less or equal to ten percent of the nominal $A R L_{0}$. Since we used a nominal $A R L_{0}$ of $500, m_{\text {opt }}$ can be expressed mathematically as

$$
m_{\text {opt }}=\operatorname{Minimum}\left\{m \mid C A A R L_{0} \approx 500 \text { and } C S D A R L_{0} \leq 50\right\}
$$

Since the Case A and Case E findings in terms of the optimal sample sizes are similar, in this section, we only display the Case E results and graphs due to space restriction. The conditional run-length distribution and its characteristics are computed using SAS ${ }^{\circledR}$ 9.4. In Fig. 4, Panels 1 and 2 display the conditional run-length distribution of the $C A R L_{0}$ and its characteristics for different Phase I sample sizes when $n=$ 5 with $\alpha=0.5$ and 1.5 , respectively. These figures show that for small Phase I sample sizes the practitioner-to-practitioner variability increases, which reveals instability in the performance of the proposed W-GWMA scheme. For instance, when $m=25$, the $C A A R L_{0}$ and $C S D A R L_{0}$ are equal to 504.09 and 95.25, respectively. However, as the Phase I sample size increases, the practitioner-to-practitioner variability decreases. For instance, when $m=100$, the $C A A R L_{0}$ and $C S D A R L_{0}$ are equal to 493.76 and 33.24, respectively (see Fig. 4 Panel 1). Table 7 gives the $C A A R L_{0}$ and $S D C A R L_{0}$ (in brackets) values for different values of $m$ for a nominal $A R L_{0}$ of 500 when $q \in\{0.1,0.9\}, \alpha \in\{0.5,1.5\}$ and $n=5$. From Table 7, it can be seen that the proposed W-GWMA scheme requires at least 50 observations to guarantee stability in the Phase II performance. In other words, the W-GWMA scheme needs at least 50 observations (i.e. $m_{o p t}=50$ ) in Case $\mathrm{U}$ to perform as if it was designed in Case $\mathrm{K}$.

Table 7

The $C A A R L_{0}$ and $C S D A R L_{0}$ values of $\mathrm{W}-\operatorname{GWMA}(q, \alpha)$ monitoring scheme

\begin{tabular}{|c|c|c|c|c|}
\hline \multirow{2}{*}{$m$} & \multicolumn{2}{|c|}{$q=0.1$} & \multicolumn{2}{|c|}{$q=0.9$} \\
\hline & $\alpha=0.5$ & $\alpha=1.5$ & $\alpha=0.5$ & $\alpha=1.5$ \\
\hline 25 & 504.09 & 523.22 & $\begin{array}{l}523.37 \\
\end{array}$ & 490.76 \\
\hline & & $(122.45)$ & & \\
\hline 40 & $\begin{array}{l}507.07 \\
(54.24)\end{array}$ & $\begin{array}{l}497.08 \\
(59.03)\end{array}$ & $\begin{array}{c}509.13 \\
(110.05)\end{array}$ & $\begin{array}{l}499.22 \\
(63.39)\end{array}$ \\
\hline & 490.14 & 504.24 & 498.99 & 498.40 \\
\hline 50 & (48.48) & $(46.25)$ & (99.99) & (56.19) \\
\hline & 503.46 & 501.39 & 498.44 & 503.11 \\
\hline 15 & $(39 . .06)$ & $(41.21)$ & (61.56) & (51.07) \\
\hline & 493.76 & 491.28 & 497.29 & 492.46 \\
\hline 100 & (33.24) & (34.64) & (53.68) & (36.27) \\
\hline & 493.63 & 495.18 & 507.15 & 498.09 \\
\hline 125 & (30.85) & (31.34) & (47.59) & (35.83) \\
\hline 150 & 501.51 & 499.23 & 504.34 & 502.10 \\
\hline 150 & $(23.85)$ & (26.74) & $(40.76)$ & (31.08) \\
\hline$m_{\text {opt }}$ & 50 & 50 & 125 & 100 \\
\hline
\end{tabular}




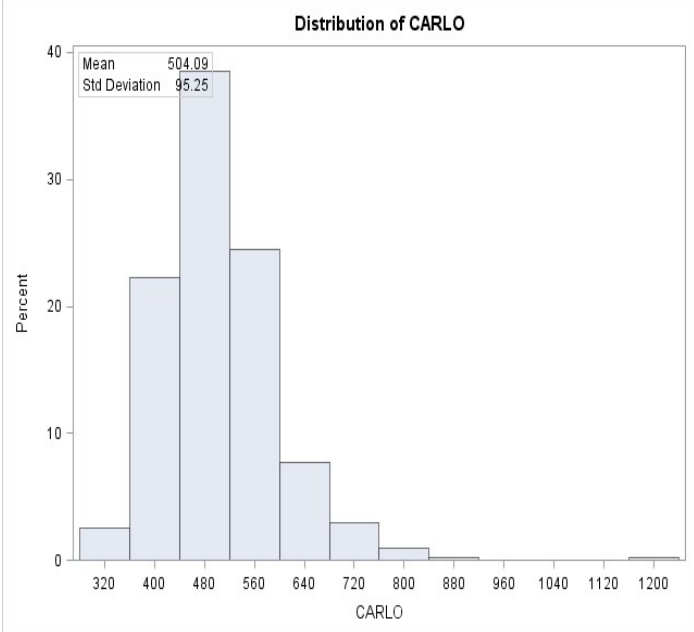

(a) $m=25, q=0.1$ and $L=2.4499$

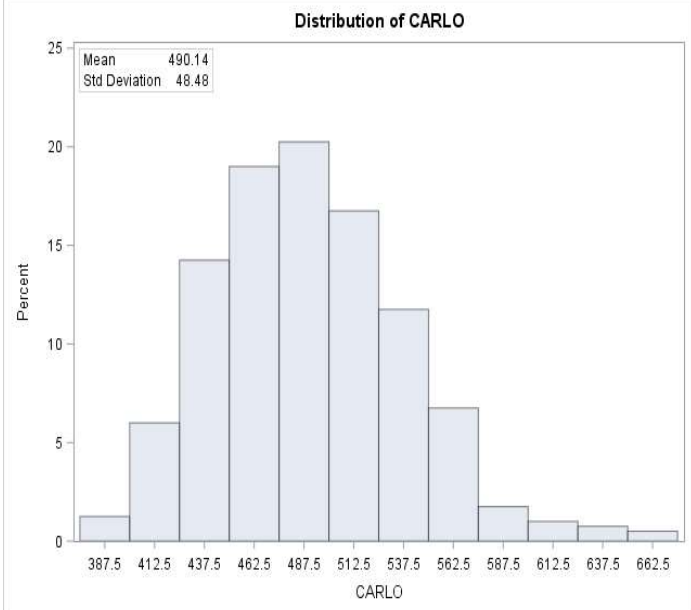

(c) $m=50, q=0.1$ and $L=2.583$

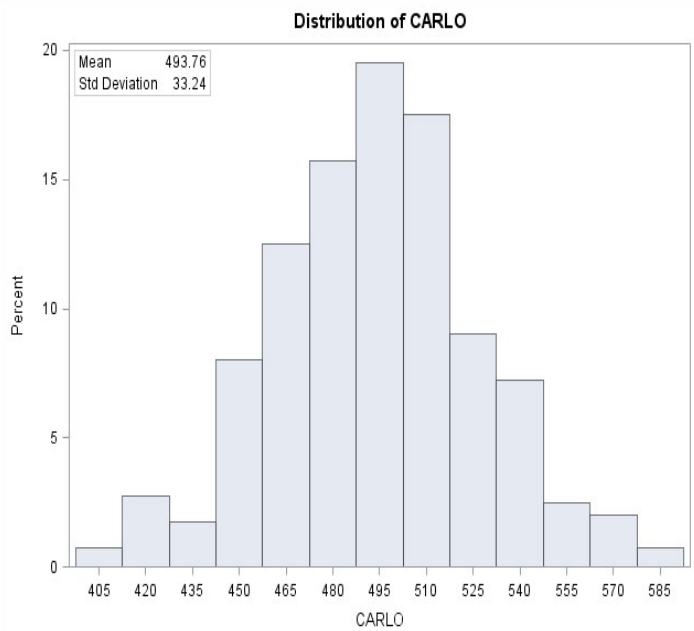

(e) $m=100, q=0.1$ and $L=3.2599$

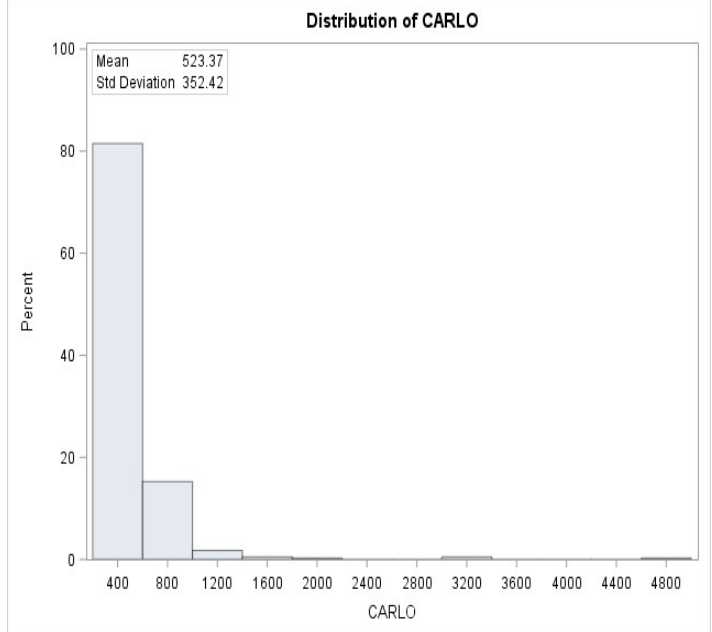

(b) $m=25, q=0.9$ and $L=2.321$

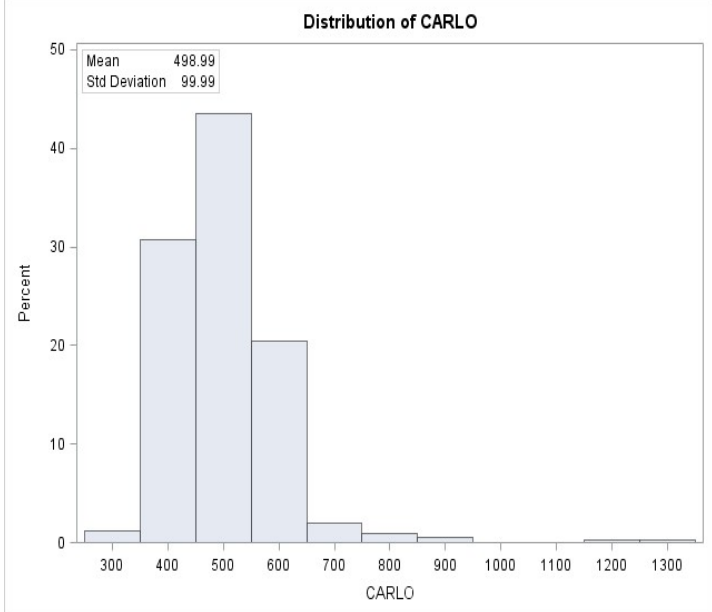

(d) $m=50, q=0.9$ and $L=2.412$

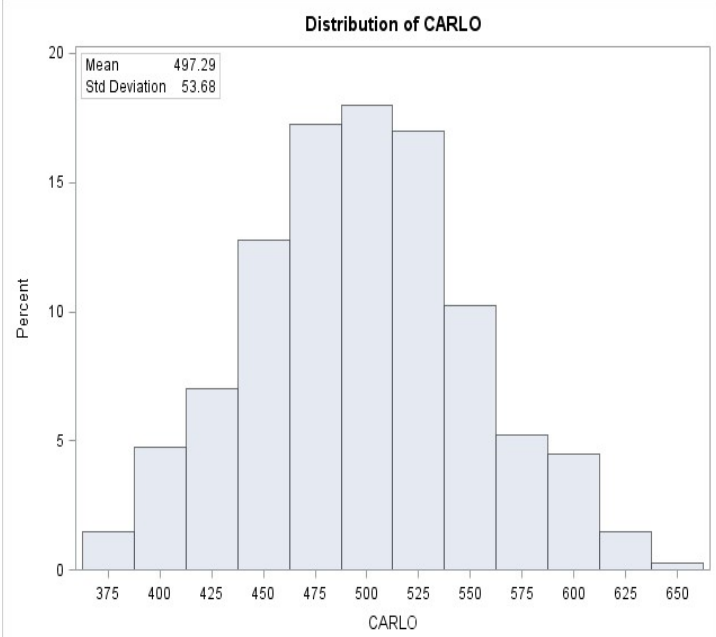

(f) $m=100, q=0.9$ and $L=2.9399$

Panel 1: $\alpha=0.5$ and $q \in\{0.1,0.9\}$ 


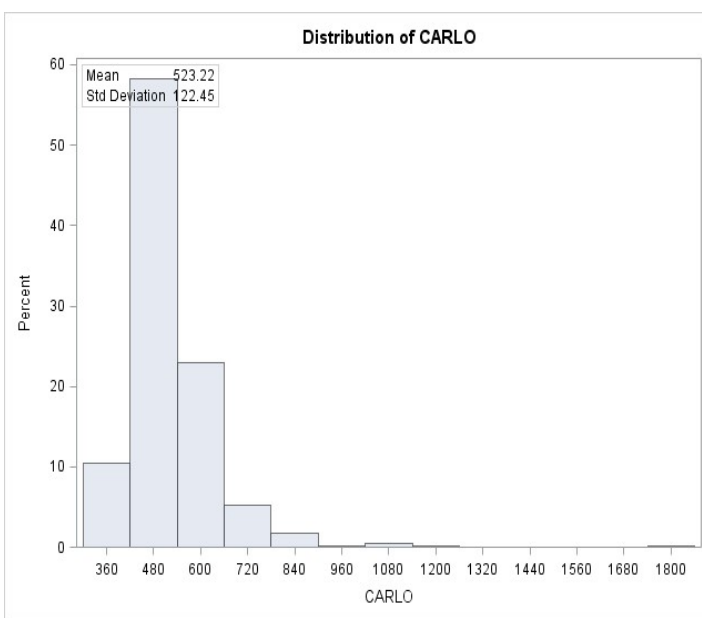

(a) $m=25, q=0.1$ and $L=2.4653$

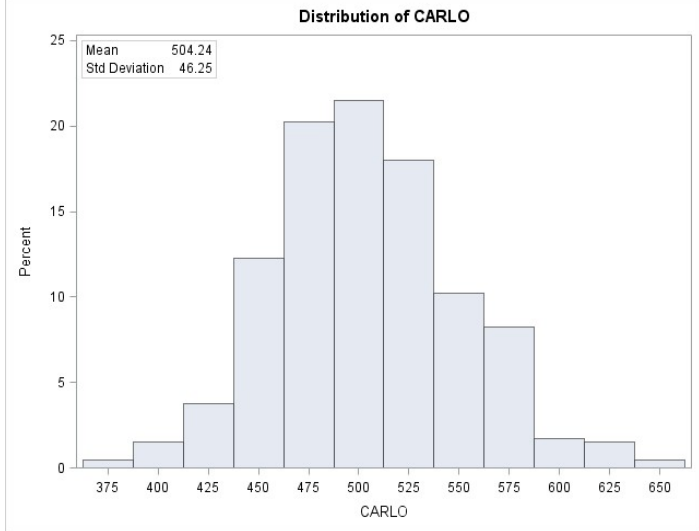

(c) $m=50, q=0.1$ and $L=3.1491$

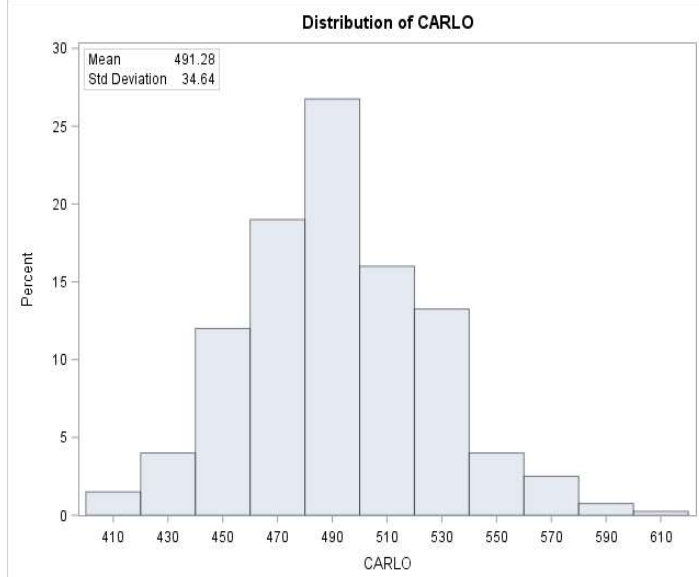

(e) $m=100, q=0.1$ and $L=3.2873$

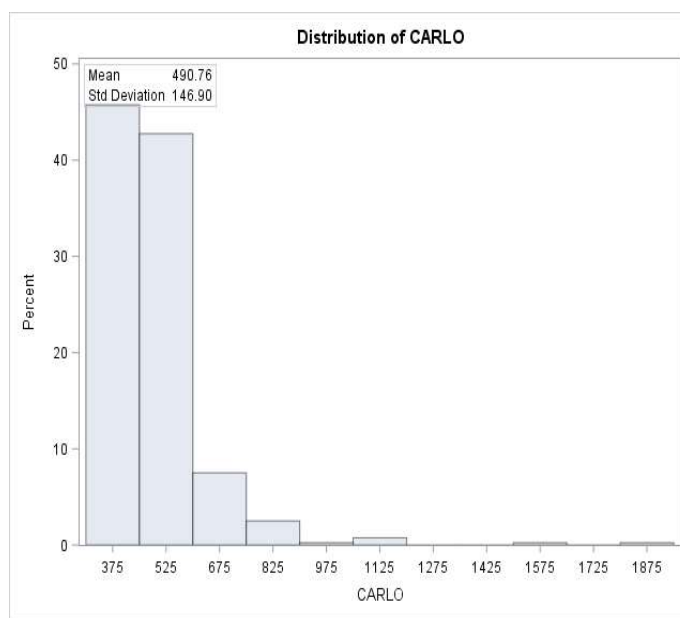

(b) $m=25, q=0.9$ and $L=2.3944$

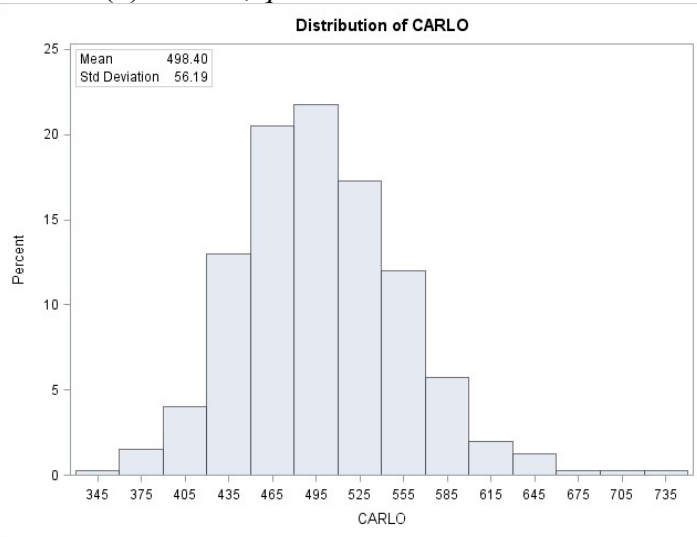

(d) $m=50, q=0.9$ and $L=2.8929$

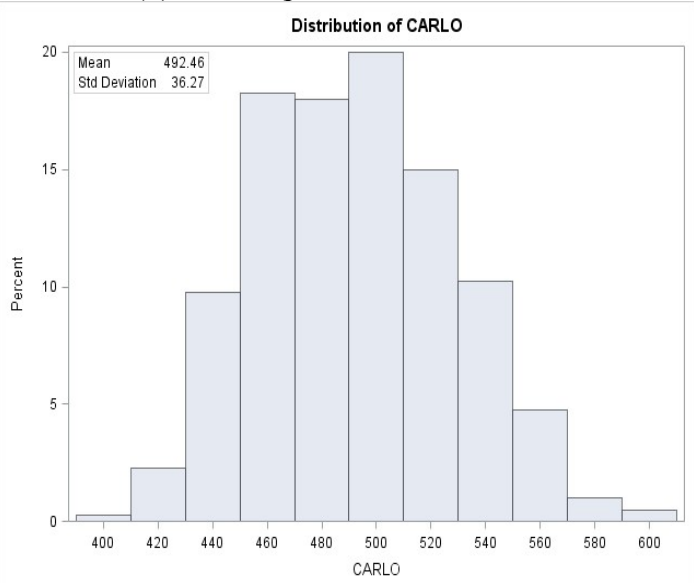

(f) $m=100, q=0.9$ and $L=2.9729$

Panel 2: $\alpha=1.5$ and $q \in\{0.1,0.9\}$

Fig. 4. Distribution of the $C A R L_{0}$ of the proposed $\operatorname{GWMA}(q, \alpha)$ when $m \in\{25,50,100\}, n=5, q \in\{0.1,0.9\}$ and $\alpha \in\{0.5,1.5\}$ in Case E.

\section{Conclusion and recommendations}

A new distribution-free GWMA monitoring scheme based on the WRS, $W$ statistic, has been proposed in order to improve and expand Li et al. (2010)'s W-EWMA scheme. The proposed scheme is more flexible than the W-EWMA scheme through an extra design parameter $\alpha$. The W-EWMA scheme is special case of the W-GWMA when $\alpha=1$. Compared to the existing schemes considered in this paper, the W-GWMA is superior in many situations and present very attractive run-length properties. Practitioners are advised to 
use the proposed monitoring scheme for small values of the design parameter $\alpha$ and large values of $q$. Moreover, whenever the underlying process distribution is unknown or non-normal, practitioners are recommended to use the proposed control scheme with at least 50 Phase I observations in order to guarantee stability in the Phase II performance.

Finally, there have been a few studies on nonparametric double GWMA (DGWMA), which combines two GWMA schemes, see for instance, Lu (2018) for the sign statistic and Karakani et al. (2019) for the exceedance statistic; hence, for future research purpose, researchers can also look at designing the DGWMA using the WRS $W$ statistic and study the effect of estimating the design parameters and Phase I sample size on the Phase II performance of the DGWMA scheme.

\section{Acknowledgement}

The authors thank the University of South Africa (UNISA) for support.

\section{References}

Adegoke, N.A., Abbasi, S.A., Dawod, A.B.A., \& Pawley, M.D.M. (2019). Enhancing the performance of the EWMA control chart for monitoring the process mean using auxiliary information. Quality and Reliability Engineering International, 35(4), 920-933.

Aslam, M., Al-Marshadi, A.H., \& Jun, C.H. (2017). Monitoring process mean using generally weighted moving average chart for exponentially distributed characteristics. Journal of Statistical Computation and Simulation, 46(5), 3712-3722.

Bag, M., Gauri, S.K., \& Chakraborty, S. (2012). Feature-based decision rules for control charts pattern recognition: A comparison between CART and QUEST algorithm. International Journal of Industrial Engineering Computations, 3(2), 199-210.

Black, G., Smith, J., \& Wells, S. (2011). The impact of Weibull data and autocorrelation on the performance of the Shewhart and exponentially weighted moving average control charts. International Journal of Industrial Engineering Computations, 2(3), 575-582.

Chakraborty, N., Chakraborti, S., Human, S.W., \& Balakrishnan, N. (2016). A generally weighted moving average signed rank control chart. Quality and Reliability Engineering International, 32(8), 2835-2845.

Chakraborty, N., Human, S.W., \& Balakrishnan, N. (2017). A generally weighted moving average chart for time between events. Communications in Statistics - Simulation and Computation, 46(10), 77907817.

Chakraborty, N., Human, S.W., \& Balakrishnan, N. (2018). A generally weighted moving average exceedance chart. Journal of Statistical Computation and Simulation, 88(9), 1759-1781.

Haq, A. (2019). A maximum adaptive exponentially weighted moving average control chart for monitoring process mean and variability. Quality Technology \& Quantitative Management, https://doi.org/ 10.1080/16843703.2018.1530181.

Haridy, S., Shamsuzzaman, M., Alsyouf, I., \& Mukherjee A. (2019). An improved design of exponentially weighted moving average scheme for monitoring attributes. International Journal of Production Research, https://doi.org/ 10.1080/00207543.2019.1605224

Jensen, W.A., Jones-Farmer, L.A., Champ, C.W., \& Woodall, W.H. (2006). Effects of parameter estimation on control chart properties: A literature review. Journal of Quality Technology, 38(4), 349364.

Karakani, H.M., Human, S.W., \& van Niekerk, J. (2019). A double generally weighted moving average exceedance control chart. Quality and Reliability Engineering International, 35(1), 224-245.

Li, S.Y., Tang, L.C., \& Ng, S.H. (2010). Nonparametric CUSUM and EWMA control charts for detecting mean shifts. Journal of Quality Technology, 42(2), 209-226.

Lim, S.A.H., Antony, J., He, Z., \& Arshed, N. (2017). Critical observations on the statistical process control implementation in the UK food industry. International Journal of Quality \& Reliability Management, 34(5), 684-700. 
Lu, S.L. (2015). An extended nonparametric exponentially weighted moving average sign control chart. Quality and Reliability Engineering International, 31(1), 3-13.

Lu, S.L. (2018). Nonparametric double generally weighted moving average sign charts based on process proportion. Communications in Statistics -Theory and Methods, 47(11), 2684-2700.

Malela-Majika, J.C., \& Rapoo, E.M. (2016). Distribution-free CUSUM and EWMA control charts based on the Wilcoxon rank-sum statistic using ranked set sampling for monitoring mean shifts. Journal of Statistical Computation and Simulation, 86(16), 3715-3734.

Montgomery, D.C. (2005). Introduction to Statistical Quality Control, 5th ed. Wiley, New York.

Roberts, S.W. (1959). Control chart tests based on geometric moving averages. Technometrics, 1(3), 239-250.

Ruggeri, F., Kenett, R.S., \& Faltin, F.W. (2007). Exponentially weighted moving average (EWMA) control chart. In Encyclopedia of Statistics in Quality and Reliability. John Wiley \& Sons: Hoboken, New Jersey, 2, 633-639.

Sheu, S.H., \& Hsieh, Y.T. (2009). The extended GWMA control chart. Journal of Applied Statistics, 36(2), 135-147.

Sheu, S.H., \& Lin, T.C. (2003). The generally weighted moving average control chart for detecting small shifts in the process mean. Quality Engineering, 16(2), 209-231.

Sheu, S.H., \& Yang, L. (2006). The generally weighted moving average control chart for monitoring the process median. Quality Engineering, 18(3), 333-344.

Shongwe, S.C., \& Graham, M.A. (2017). Synthetic and runs-rules charts combined with an $\bar{X}$ chart: Theoretical discussion. Quality and Reliability Engineering International, 35(1), 7-35.

Simoglou, A, Martin, E.B., Morris, A.J., Wood, M., \& Jones, G.C. (1997). Multivariate statistical process control in chemicals manufacturing. International Federation of Automatic Control (IFAC) Proceedings Volumes, 30(18), 21-28.

Sukparungsee, S. (2018). Robustness of the generally weighted moving average signed-rank control chart for monitoring a shift of skew processes. Matter: International Journal of Science and Technology, 4(3), https://doi.org/10.20319/mijst.2018.43.125137.

Tai, S., Lin, C., \& Chen, Y. (2009). Design and implementation of the extended exponentially weighted moving average control charts. In Proceedings of the 2009 International Conference on Management and Service Science, IEEE Xplore, (MASS) (October 2009) pp. 1-4.

Teh, S.Y., Khoo, M.B.C., \& Wu, Z. (2012). Monitoring process mean and variance with a single generally weighted moving average chart. Communications in Statistics -Theory and Methods, 41(12), 2221-2241.

Teh, S.Y., Khoo, M.B.C., Hong, K.H., \& Soh, K.L. (2014). A comparative study of the median run length (MRL) performance of the Max-DEWMA and SS-DEWMA control charts. Proceedings of the 2014 International Conference on Industrial Engineering and Operations Management, Bali, Indonesia, January.

Wilcoxon, F., Individual comparisons by ranking methods, Biometrics, 1(6), 80-83.

Zhang, M., Megahed, F.M., \& Woodall, W.H. (2014). Exponential CUSUM charts with estimated control limits. Quality and Reliability Engineering International, 30(2), 275-286.

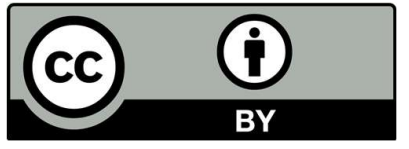

(C) 2020 by the authors; licensee Growing Science, Canada. This is an open access article distributed under the terms and conditions of the Creative Commons Attribution (CCBY) license (http://creativecommons.org/licenses/by/4.0/). 